\title{
Synthesis and antibacterial activities of acylide derivatives bearing an aryl-tetrazolyl chain
}

This article was published in the following Dove Press journal:

Drug Design, Development and Therapy

24 September 2014

Number of times this article has been viewed

\author{
Ling-Xing Shan' \\ Ping-Hua Sun ${ }^{1,2}$ \\ Bao-Qin Guo' \\ Xing-Jun $\mathrm{Xu}{ }^{\prime}$ \\ Zhi-Qiang $\mathrm{Li}^{\prime}$ \\ Jia-Zhi Sun ${ }^{2}$ \\ Shu-Feng Zhou ${ }^{2}$ \\ Wei-Min Chen' \\ 'Guangdong Province Key Laboratory \\ of Pharmacodynamic Constituents \\ of Traditional Chinese Medicine and \\ New Drugs Research, College of \\ Pharmacy, Jinan University, \\ Guangzhou, People's Republic of \\ China; ${ }^{2}$ College of Pharmacy, University \\ of South Florida, Tampa, FL, USA
}

Correspondence: Ping-Hua Sun Guangdong Province Key Laboratory of Pharmacodynamic Constituents of TCM and New Drugs Research, College of Pharmacy, Jinan University, Guangzhou 510632,

People's Republic of China

Tel $+86208522 \quad 1367$

Fax +86208522 4766

Email pinghuasunny@163.com

Wei-Min Chen

Guangdong Province Key

Laboratory of Pharmacodynamic

Constituents of TCM and New

Drugs Research, College of Pharmacy,

Jinan University, Guangzhou 5I0632,

People's Republic of China

Tel +86 $208522 \quad$ I 367

Fax +86 2085224766

Email twmchen@jnu.edu.cn

\begin{abstract}
Seventeen acylides bearing an aryl-tetrazolyl alkyl-substituted side chain were synthesized, starting from clarithromycin, via several reactions including hydrolysis, acetylating, esterification, carbamylation, and Michael addition. The structures of all new compounds were confirmed by ${ }^{1} \mathrm{H}$ nuclear magnetic resonance spectroscopy, ${ }^{13} \mathrm{C}$ nuclear magnetic resonance spectroscopy, and mass spectrometry. All these synthesized acylides were evaluated for in vitro antimicrobial activities against gram-positive pathogens (Staphylococcus aureus, Staphylococcus epidermidis) and gram-negative pathogens (Pseudomonas aeruginosa, Escherichia coli), using the broth microdilution method. Results showed that compounds 10e, 10f, 10g, $10 \mathrm{~h}, 10 \mathrm{o}$ have good antibacterial activities.
\end{abstract}

Keywords: acylide, clarithromycin, synthesis, antibacterial activity

\section{Introduction}

Since the 1950s, macrolide antibiotics have been widely used for the clinical treatment of respiratory tract or soft tissue infections, but because of the extensive use of antibiotics, bacterial resistance has increased year by year. ${ }^{1-4}$ Allen proved that cladinose is the key group for bacterial resistance, and deglycosylation or modification of its cladinose is a feasible way to change a drug's resistance. ${ }^{5}$ Modification at C-3 could enhance activity against efflux resistance. Tanikawa et al synthesized a series of clarithromycin derivatives in which hydrolysis and acylation occur at the C-3 position. ${ }^{6}$ It has been proven that compounds TEA-0769 and FMA-481 have better antibacterial activity than clarithromycin for macrolide-susceptible strains. They also have antibacterial activity against MLS $_{\text {B }}$-resistant Staphylococcus aureus and efflux-resistant Streptococcus pneumoniae. After ketolide, acylide is another class of erythromycin derivatives that has antibacterial activity against macrolide resistance strains and has the potential to become a new generation of macrolide antibiotics.

Tetrazole, a bioisostere of carboxyl group or a peptide bond, ${ }^{7,8}$ is an important pharmacophore widely used in drugs such as matrix metalloproteinase inhibitors, ${ }^{9}$ monoamine oxidase B inhibitors, ${ }^{10}$ and antibacterial, antifungal, and antiproliferative agents. ${ }^{11}$ Water solubility can be improved by introducing tetrazole, because of its hydrophility, and its basicity will be also increased. Therefore, the introduction of tetrazole into acylide may not only enhance antibacterial activity but also improve its pharmacokinetic properties. On the basis of the previous study about ketolides, performed in our laboratory, ${ }^{12}$ the cladinosyl of the C-3 position in macrolide was substituted by aroyl, and the carbamate ring was connected with some different alky side chains bearing aryl-tetrazolyl. As a consequence, 17 new acylide derivatives 
were synthesized and evaluated for in vitro antimicrobial activities.

\section{Materials and methods Chemistry}

Unless otherwise noted, all materials were commercially available and used without further purification. Dichloromethane and triethylamine were distilled from calcium hydride and sodium, respectively. The reactions were monitored by thin-layer chromatography (silica gel 60 GF254; Qingdao Haiyang Chemical Co, Ltd, Qingdao, Shandong, People's Republic of China) at $254 \mathrm{~nm} .{ }^{1} \mathrm{H}$ nuclear magnetic resonance spectroscopy (NMR) and ${ }^{13} \mathrm{C}$ NMR spectra were recorded on Bruker-AV300MHz nuclear magnetic resonance spectrometers (Bruker Biosciences Corporation, Billerica, MA, USA). Chemical shifts are provided in parts per million downfield from tetramethylsilane (internal standard) with a coupling constant in hertz. Mass spectra and high-resolution mass spectrometry (HRMS) results were recorded on Thermo Finnigan LCQ Advantage MAX (Thermo Fisher Scientific, Waltham, MA, USA).

The compounds 2-(2H-tetrazol-5-yl) pyridine (3a), 3-(2H-tetrazol-5-yl) pyridine (3b), 4-(2H-tetrazol-5-yl) pyridine (3c), 5-phenyl-2H-tetrazole (3d), and 5-(thiophen-2-yl)-2Htetrazole $(3 \mathrm{e})$ were prepared by a known procedure, ${ }^{13}$ and 1-methyl-1H-tetrazole-5-thiol (3f) was commercially available. The compounds $4 a-4 q$ and $5 a-5 q$ have been synthesized via a method we previously published. ${ }^{12}$

\section{General procedure for preparation}

of compounds $4 a-4 q$

To a solution of compounds $3 \mathrm{a}-3 \mathrm{f}(1 \mathrm{mmol})$ in anhydrous $\mathrm{N}$, N-dimethyl formamide ( $5 \mathrm{~mL}$ ) were added, successively, N-bromo-phthalimide (1 mmol) and anhydrous potassium carbonate $(1 \mathrm{mmol})$, and the mixture was stirred for 10 hours at $80^{\circ} \mathrm{C}$ under nitrogen. The reaction mixture was quenched with ice water $(10 \mathrm{~mL})$. A crystalline solid was precipitated, and the filtered product was purified by silica-gel column chromatography (ether/ethyl acetate acetate $=1: 1$ to petroleum ether/ethyl acetate/dichloromethane $=1: 1: 1$ ) to afford $4 \mathrm{a}-4 \mathrm{q}$ as white solid.

\section{General procedure for the synthesis} of compounds $5 \mathrm{a}-5 \mathrm{q}$

To a solution of compounds $4 \mathrm{a}-4 \mathrm{q}(1 \mathrm{mmol})$ in the mixture of anhydrous ethanol $(7 \mathrm{~mL})$ and acetonitrile $(5 \mathrm{~mL})$ was added hydrazine monohydrate $(2 \mathrm{mmol})$, and the mixture was then refluxed for 6 hours. After cooling, the mixture was filtered and the filtrate was evaporated in vacuo. Aqueous $\mathrm{NaOH}(20 \mathrm{mmol})$ was subsequently added to the residue and then extracted with dichloromethane (10 $\mathrm{mL}$ three times), washed with a saturated brine, dried over anhydrous sodium sulfate, filtered, and concentrated in vacuo. The purification of the crude product by silica gel column chromatography (dichloromethane $/$ methanol/triethylamine $=9: 1: 0.1$ ) afforded the desired compounds $5 \mathrm{a}-5 \mathrm{q}$.

\section{3-O-descladinosyl-10, I I-anhydro-6-O-methyl- erythromycin A (compound 6)}

To a solution of ethylene carbonate $(51.6 \mathrm{mmol})$ and triethylamine $(40 \mathrm{~mL})$, clarithromycin $(13.4 \mathrm{mmol})$ was added, and the reaction mixture was refluxed for 24 hours. Then additional ethylene carbonate $(34.4 \mathrm{mmol})$ was added and the mixture was refluxed for 18 more hours. The solvent was removed under reduced pressure, and $23 \mathrm{mmol} \mathrm{HCl}$ aqueous solution, $40 \mathrm{~mL}$ ethanol, and $100 \mathrm{~mL}$ water were added. The reaction mixture was stirred for 24 hours at room temperature and then basified with $\mathrm{NaOH}$ aqueous solution ( $1 \mathrm{~mol} / \mathrm{L})$ to $\mathrm{Ph} 10-11$. The precipitated, crude product was filtered off and recrystallized from the mixture of petroleum ether and acetone to afford compound 6, a white solid (7.2 g, 94.1\%). ${ }^{1} \mathrm{H} \mathrm{NMR}\left(300 \mathrm{MHz}, \mathrm{CDCl}_{3}\right) \delta 6.39$ (s, 1H), $5.00(\mathrm{~d}, \mathrm{~J}=9.1 \mathrm{~Hz}, 1 \mathrm{H}), 4.69$ (d, J=5.0 Hz, 2H), 3.85 (s, 1H), $3.74(\mathrm{t}, \mathrm{J}=9.3 \mathrm{~Hz}, 1 \mathrm{H}), 3.49$ (dd, J=10.8, $5.5 \mathrm{~Hz}, 1 \mathrm{H}), 3.15$ (dd, $\mathrm{J}=27.9,12.8 \mathrm{~Hz}, 1 \mathrm{H}), 3.08(\mathrm{~s}, 3 \mathrm{H}), 2.74-2.56(\mathrm{~m}, 3 \mathrm{H})$, $2.44(\mathrm{~s}, 3 \mathrm{H}), 2.22(\mathrm{~s}, 6 \mathrm{H}), 2.09(\mathrm{~s}, 3 \mathrm{H}), 2.02(\mathrm{~s}, 3 \mathrm{H}), 1.94$ $(\mathrm{dd}, \mathrm{J}=9.2,4.9 \mathrm{~Hz}, 1 \mathrm{H}), 1.88-1.77(\mathrm{~m}, 1 \mathrm{H}), 1.72(\mathrm{dd}, \mathrm{J}=12.9$, $2.6 \mathrm{~Hz}, 1 \mathrm{H}), 1.52$ (dd, J=12.7, $6.6 \mathrm{~Hz}, 3 \mathrm{H}), 1.37$ (s, 3H), 1.27 (d, J=3.9 Hz, 6H), 1.16 (d, J=6.3 Hz, 3H), 0.91 (s, 3H), 0.88 (s, 3H). ${ }^{13} \mathrm{C} \mathrm{NMR}\left(75 \mathrm{MHz}, \mathrm{CDCl}_{3}\right) \delta 207.59,176.68,141.29$, $138.77,106.77,91.96,80.90,79.17,77.65,77.22,73.63$, $70.50,69.69,65.58,48.26,44.33,40.29,38.32,36.93,36.40$, $30.93,21.34,20.86,20.37,20.22,16.13,15.88,12.94$, $10.50,7.62$.

2'-Acetyl-3-O-descladinosyl - 10, I I -anhydro-6-Omethyl-erythromycin A (compound 7)

To a solution of acetic anhydride $(16.8 \mathrm{mmol})$ and anhydrous triethylamine $(16.8 \mathrm{mmol})$ in dichloromethane $(30 \mathrm{~mL})$, compound $\mathbf{6}(8.4 \mathrm{mmol})$ was added and stirred for 24 hours at room temperature. The reaction mixture was poured into saturated aqueous $\mathrm{NaHCO}_{3}(30 \mathrm{~mL})$ and extracted with $\mathrm{CH}_{2} \mathrm{Cl}_{2}$ $(20 \mathrm{~mL} \times 3)$. The organic layer was washed with brine, dried over anhydrous $\mathrm{Na}_{2} \mathrm{SO}_{4}$, and filtered, and the solvent was removed under reduced pressure to yield white solid 7 (4.68 g, $90 \%$ ). ${ }^{1} \mathrm{HNMR}\left(300 \mathrm{MHz}, \mathrm{CDCl}_{3}\right) \delta 6.40(\mathrm{~s}, 1 \mathrm{H}), 5.28(\mathrm{~s}, 1 \mathrm{H})$, 
5.07-4.93 (m, 1H), $4.68(\mathrm{dd}, \mathrm{J}=9.5,5.3 \mathrm{~Hz}, 2 \mathrm{H}), 3.86(\mathrm{~d}, \mathrm{~J}=2.7$ $\mathrm{Hz}, 1 \mathrm{H}), 3.76$ (d, J=10.4 Hz, 1H), 3.49 (dd, J=9.4, $5.9 \mathrm{~Hz}, 1 \mathrm{H}$ ), 3.12 (d, J=7.0 Hz, 1H), 3.08 (s, 2H), 2.63 (dd, J=10.4, $6.8 \mathrm{~Hz}$, 2H), 2.18 (d, J=17.9 Hz, 6H), 2.08 (s, 3H), 2.02 (s, 2H), 1.92 (dd, J=14.7, 7.1 Hz, 1H), 1.84-1.65 (m, 2H), 1.64-1.41 (m, $3 \mathrm{H}), 1.37(\mathrm{~d}, \mathrm{~J}=7.7 \mathrm{~Hz}, 3 \mathrm{H}), 1.33-1.18(\mathrm{~m}, 12 \mathrm{H}), 1.16(\mathrm{~d}, \mathrm{~J}=6.5$ $\mathrm{Hz}, 3 \mathrm{H}), 0.93-0.82(\mathrm{~m}, 6 \mathrm{H}) .{ }^{13} \mathrm{C} \mathrm{NMR}\left(75 \mathrm{MHz}, \mathrm{CDCl}_{3}\right) \delta$ 207.80, 175.83, 170.25, 140.93, 139.43, 102.53, 79.97, 79.38, $77.15,73.29,71.80,69.18,64.09,48.84,44.26,40.69,37.69$, $37.43,37.18,30.12,29.69,21.45,21.29,21.19,20.66,20.27$, $17.00,15.55,13.51,10.58,7.94$.

\section{2'-Acetyl-3-O-descladinosyl-3-O-(3-pyridyl)acetyl-10, I I-anhydro-6-O-methyl-erythromycin A (compound 8)}

Triethylamine $(10.8 \mathrm{mmol})$ was dissolved in a solution of 3-pyridine acetic acid hydrochloride $(10.8 \mathrm{mmol})$ in anhydrous dichloromethane $(20 \mathrm{~mL})$. The mixture was cooled to $-15^{\circ} \mathrm{C}$, and pivaloyl chloride $(21.6 \mathrm{mmol})$ was added drop wise, with intensive stirring under nitrogen. After completion of dripping, the resulting reaction mixture was stirred for 1 hour at $-15^{\circ} \mathrm{C}$, followed by the drop-wise addition of a solution of compound 7 (3.2 $\mathrm{mmol})$ in anhydrous dichloromethane $(10 \mathrm{~mL})$ over a period of 15 minutes. 4-Dimethylaminopyridine $(0.32 \mathrm{mmol})$ was then added and stirred for 5 hours at room temperature. The reaction mixture was finally poured into saturated aqueous $\mathrm{NaHCO}_{3}(30 \mathrm{~mL})$ and extracted with dichloromethane. The organic layer was washed with brine, dried over anhydrous sodium sulfate, filtered, and evaporated in vacuo. The crude product was purified by column chromatography (petroleum ether/acetone/triethylamine $=6: 1: 1$ to $4: 1: 1)$ to afford the desired product 8 as white solid (1.6 g, 66.8\%). NMR $\left(300 \mathrm{MHz} \mathrm{CDCl}_{3}\right) \delta 8.55-8.44(\mathrm{~m}, 2 \mathrm{H}), 7.66(\mathrm{dt}, \mathrm{J}=7.9$, $1.9 \mathrm{~Hz}, 1 \mathrm{H}), 7.32-7.21(\mathrm{~m}, 2 \mathrm{H}), 6.55(\mathrm{~d}, \mathrm{~J}=0.9 \mathrm{~Hz}, 1 \mathrm{H})$, $5.58(\mathrm{dd}, \mathrm{J}=5.3,1.8 \mathrm{~Hz}, 1 \mathrm{H}), 5.05(\mathrm{dd}, \mathrm{J}=10.8,2.5 \mathrm{~Hz}$, $1 \mathrm{H}), 4.66(\mathrm{dd}, \mathrm{J}=10.5,7.5 \mathrm{~Hz}, 1 \mathrm{H}), 4.17(\mathrm{~d}, \mathrm{~J}=7.4 \mathrm{~Hz}$, $1 \mathrm{H}), 3.65$ (s, 2H), 3.43 (d, J=7.6 Hz, 1H), 3.31 (dd, J=10.1, $6.2 \mathrm{~Hz}, 1 \mathrm{H}), 3.03(\mathrm{~s}, 2 \mathrm{H}), 2.86(\mathrm{~s}, 3 \mathrm{H}), 2.66-2.55(\mathrm{~m}, 3 \mathrm{H})$, $2.23(\mathrm{~s}, 6 \mathrm{H}), 2.10$ (d, J=3.9 Hz, 1H), 2.04 (s, 3H), 1.92 (d, J=0.6 Hz, 3H), 1.83 (ddd, J=14.1, 7.5, 2.6 Hz, 1H), 1.69 (dd, J=13.0, 2.5 Hz, 1H), 1.47 (ddd, J=14.1, 10.7, 7.3 Hz, $2 \mathrm{H}), 1.34(\mathrm{~s}, 3 \mathrm{H}), 1.23(\mathrm{~s}, 3 \mathrm{H}), 1.20-1.17(\mathrm{~m}, 6 \mathrm{H}), 0.99$ (d, J=7.1 Hz, 3H), 0.94 (d, J=7.0 Hz, 3H), 0.88 (t, J=7.4 Hz, $3 \mathrm{H}) .{ }^{13} \mathrm{C} \mathrm{NMR}\left(75 \mathrm{MHz}, \mathrm{CDCl}_{3}\right) \delta 206.94,173.49,172.39$, $169.82,150.25,148.64,139.73,137.12,129.28,123.50$, $101.78,93.47,82.62,79.39,77.23,75.96,73.99,71.38$, $70.43,69.12,63.12,49.97,42.41,40.81,40.42,40.16$,
$38.37,31.78,30.50,27.31,23.09,22.17,21.39,21.17$, $20.99,20.41,19.72,13.52,12.76,10.70,10.14$.

\section{2'-Acetyl-3-O-descladinosyl-3-O-(3-pyridyl) acetyl-I0, I I-anhydro-I I-deoxy-I 2-O-(IH-I- imidazoylcarbonyl)-6-O-methyl-erythromycin A (compound 9)}

Compound 8 (1.4 mmol), carbonyl diimidazole (5.6 mmol), and 4-dimethylamino pyridine $(0.14 \mathrm{mmol})$ were dissolved in anhydrous dichloromethane $(20 \mathrm{~mL})$. The solution was stirred for 48 hours at room temperature. The reaction mixture was quenched with saturated aqueous $\mathrm{NaHCO}_{3}(20 \mathrm{~mL})$ and extracted with dichloromethane. The organic layer was washed with saturated brine, dried over anhydrous sodium sulfate, filtered, and concentrated under reduced pressure. The crude product was recrystallized from the mixture of petroleum ether and acetone (4:1) to afford white solid $9(0.82$ g, 80\%). ${ }^{1} \mathrm{H}$ NMR (300 MHz, $\left.\mathrm{CDCl}_{3}\right) \delta 8.60-8.53(\mathrm{~m}, 2 \mathrm{H})$, $8.06(\mathrm{~s}, 1 \mathrm{H}), 7.77-7.70(\mathrm{~m}, 1 \mathrm{H}), 7.38-7.27(\mathrm{~m}, 2 \mathrm{H}), 7.05(\mathrm{dd}$, $\mathrm{J}=1.6,0.8 \mathrm{~Hz}, 1 \mathrm{H}), 6.64(\mathrm{~s}, 1 \mathrm{H}), 5.84(\mathrm{dd}, \mathrm{J}=10.2,3.0 \mathrm{~Hz}, 1 \mathrm{H})$, 5.10 (d, J=10.3 Hz, 1H), 4.65 (dd, J=10.5, 7.4 Hz, 1H), 3.90 (s, 1H), $3.70(\mathrm{~s}, 2 \mathrm{H}), 3.60(\mathrm{~d}, \mathrm{~J}=4.8 \mathrm{~Hz}, 1 \mathrm{H}), 3.11(\mathrm{~s}, 3 \mathrm{H})$, 3.08-3.02 (m, 1H), $2.86(\mathrm{~d}, \mathrm{~J}=5.9 \mathrm{~Hz}, 1 \mathrm{H}), 2.65-2.52(\mathrm{~m}$, 1H), 2.24 (s, 6H), 2.15 (s, 1H), 2.04 (s, 3H), 1.85 (s, 3H), $1.77(\mathrm{~s}, 3 \mathrm{H}), 1.70-1.61(\mathrm{~m}, 2 \mathrm{H}), 1.35$ (dd, J=9.8, $4.8 \mathrm{~Hz}, 2 \mathrm{H})$, 1.23 (s, 3H), 1.20 (d, J=4.1 Hz, 3H), 1.13 (dd, J=6.1, $3.9 \mathrm{~Hz}$, 6H), 1.01 (d, J=6.7 Hz, 3H), 0.89 (d, J=1.5 Hz, 3H), 0.86 $(\mathrm{d}, \mathrm{J}=5.3 \mathrm{~Hz}, 3 \mathrm{H}) .{ }^{13} \mathrm{C} \mathrm{NMR}\left(75 \mathrm{MHz}, \mathrm{CDCl}_{3}\right) \delta 170.19$, $169.80,150.24,148.85,145.84,137.63,137.08,130.82$, $129.34,123.60,117.13,100.99,84.37,78.35,75.82,71.16$, $69.13,63.21,50.60,42.90,40.52,38.50,30.28,29.69,27.42$, 21.44, 20.89, 19.92, 15.11, 13.57, 10.26.

\section{General procedure for the synthesis of compounds $10 \mathrm{a}-10 \mathrm{q}$}

To a solution of compound $9(0.30 \mathrm{mmol})$ in acetonitrile $(1 \mathrm{~mL})$, compounds $5 \mathrm{a}-5 \mathrm{q}(0.60 \mathrm{mmol})$ were added, and the resulting mixture was stirred for $16-25$ hours at $55^{\circ} \mathrm{C}$. After cooling, $5 \% \mathrm{KH}_{2} \mathrm{PO}_{4}(2 \mathrm{~mL})$ was added. The mixture was extracted with ethyl acetate ester. The organic layer was washed with brine, dried over anhydrous sodium sulfate, and concentrated under reduced pressure. The resulting residue was dissolved in methanol $(1 \mathrm{~mL})$, and the solution was refluxed for 25 hours. The solvent was removed under reduced pressure, and the crude product was purified by column chromatography (petroleum ether/acetone/ triethylamine $=5: 1: 1$ ) to afford compounds $10 \mathrm{a}-10 \mathrm{q}$ as white foam. 


\section{Characterization of compounds $10 \mathrm{a}-10 \mathrm{q}$,} of which some solid or powder compounds like 10c, 10d, 10g, 10k, I0n, 100 , and $10 q$ give melting point data 3-O-descladinosyl-3-O-(3-pyridyl)acetyl-6-Omethyl- I I, I -dideoxy- I 2, I I-(oxycarbony|[(3[5-(pyridin-2-yl)-I H-tetrazol-I-yl])propyl]imino) erythromycin $\mathrm{A}$ (compound I0a)

Yield 53.7\%. ${ }^{1} \mathrm{H}$ NMR ( $\left.300 \mathrm{MHz}, \mathrm{CDCl}_{3}\right) \delta 8.69$ (dd, J=4.8, $0.7 \mathrm{~Hz}, 1 \mathrm{H}), 8.55-8.42(\mathrm{~m}, 2 \mathrm{H}), 8.27$ (d, J=7.9 Hz, 1H), $7.82(\mathrm{td}, \mathrm{J}=7.8,1.7 \mathrm{~Hz}, 1 \mathrm{H}), 7.70(\mathrm{~d}, \mathrm{~J}=7.9 \mathrm{~Hz}, 1 \mathrm{H}), 7.37$ (ddd, J=7.6, 4.9, $1.0 \mathrm{~Hz}, 1 \mathrm{H}), 7.25$ (dd, J=7.9, $4.7 \mathrm{~Hz}, 1 \mathrm{H}$ ), $5.26(\mathrm{~s}, 3 \mathrm{H}), 5.10-4.97(\mathrm{~m}, 2 \mathrm{H}), 4.96-4.88(\mathrm{~m}, 2 \mathrm{H}), 3.81$ (d, J=7.2 Hz, 1H), 3.67-3.61 (m, 3H), 3.13 (dd, J=10.1, $7.2 \mathrm{~Hz}, 1 \mathrm{H}$ ), 3.05-2.94 (m, 2H), 2.85 (s, 3H), 2.82-2.74 (m, 1H), 2.41 (ddd, J=20.6, 12.6, 5.9 Hz, 3H), 2.28 (s, 6H), $2.10(\mathrm{~s}, 3 \mathrm{H}), 1.90-1.76(\mathrm{~m}, 1 \mathrm{H}), 1.58-1.47$ (m, 3H), 1.33 (s, 3H), 1.19 (d, J=5.2 Hz, 3H), $1.12(\mathrm{~s}, 6 \mathrm{H}), 1.08$ (s, 3H), 1.07 (d, J=6.4 Hz, 4H), 1.03 (d, J=7.1 Hz, 3H), 0.93 (d, J=6.8 $\mathrm{Hz}, 3 \mathrm{H}), 0.83$ (d, J=6.7 Hz, 3H), 0.71 (t, J=7.3 Hz, 3H). ${ }^{13} \mathrm{C}$ NMR $\left(75 \mathrm{MHz}, \mathrm{CDCl}_{3}\right) \delta 215.55,174.06,170.49$, 157.22, 151.75, 150.06, 149.55, 148.51, 144.89, 137.37, $129.52,125.22,124.46,123.59,103.68,82.84,81.07,78.34$, $77.58,77.16,76.74,76.38,70.12,69.22,65.80,60.73,49.97$, 47.62, 45.47, 42.87, 41.54, 39.97, 38.71, 38.32, 36.32, 30.93, 29.64, 28.51, 27.57, 21.94, 20.98, 19.52, 18.74, 15.01, 14.15, 10.11, 8.87. HRMS (ESI) m/z calcd for $\mathrm{C}_{47} \mathrm{H}_{68} \mathrm{~N}_{8} \mathrm{O}_{11}$ $[\mathrm{M}+\mathrm{H}]^{+}:$921.5080, found: 921.5081 .

3-O-descladinosyl-3-O-(3-pyridyl)acetyl-6-Omethyl- I I, I 2-dideoxy- I 2, I I-(oxycarbony|[(3[5-(pyridin-2-yl)-2H-tetrazol-2-yl])propyl]imino) erythromycin A (compound IOb)

Yield 54.6\%. ${ }^{~} \mathrm{H}$ NMR (300 MHz, $\left.\mathrm{CDCl}_{3}\right) \delta 8.70(\mathrm{~d}, \mathrm{~J}=4.2 \mathrm{~Hz}$, $1 \mathrm{H}), 8.53-8.41(\mathrm{~m}, 2 \mathrm{H}), 8.17$ (d, J=7.9 Hz, 1H), 7.77 (td, J=7.8, $1.7 \mathrm{~Hz}, 1 \mathrm{H}), 7.70$ (d, J=7.9 Hz, 1H), 7.36-7.21 (m, 2H), $5.48(\mathrm{~s}, 3 \mathrm{H}), 5.04-4.87(\mathrm{~m}, 2 \mathrm{H}), 4.84-4.67(\mathrm{~m}, 2 \mathrm{H}), 3.81$ (d, J=7.1 Hz, 1H), 3.66 (dd, J=8.0, $3.9 \mathrm{~Hz}, 3 \mathrm{H}), 3.14$ (dd, J=9.7, $6.9 \mathrm{~Hz}, 1 \mathrm{H}), 3.06-2.92(\mathrm{~m}, 2 \mathrm{H}), 2.91(\mathrm{~s}, 3 \mathrm{H}), 2.48-2.38$ (m, 3H), 2.29 (s, 6H), 2.09 (s, 3H), 1.86 (dd, J=11.1, 6.2 $\mathrm{Hz}, 1 \mathrm{H}), 1.56-1.46$ (m, 3H), 1.35 (s, 3H), 1.17 (d, J=2.9 Hz, $3 \mathrm{H}), 1.12$ (s, 6H), 1.08 (d, J=5.3 Hz, 3H), 1.02 (s, 3H), 0.94 $(\mathrm{d}, \mathrm{J}=6.7 \mathrm{~Hz}, 3 \mathrm{H}), 0.85$ (d, J=6.7 Hz, 3H), 0.77 (t, J=7.3 Hz, 3H). ${ }^{13} \mathrm{C} \mathrm{NMR}\left(75 \mathrm{MHz}, \mathrm{CDCl}_{3}\right) \delta 215.68,174.15,170.52,164.66$, 157.28, 150.08, 148.44, 146.85, 137.47, 137.05, 129.55, 124.69 , 123.60, 122.46, 103.65, 82.90, 81.07, 78.34, 76.53, 70.08, 69.12, $65.79,60.56,51.20,50.18,45.47,42.88,41.36,39.91,38.73$, $38.29,36.37,30.92,29.64,28.58,27.52,27.04,21.96,20.95$,
19.44, 18.75, 15.02, 14.19, 10.15, 8.89. HRMS (ESI) m/z calcd for $\mathrm{C}_{47} \mathrm{H}_{68} \mathrm{~N}_{8} \mathrm{O}_{11}[\mathrm{M}+\mathrm{H}]^{+}:$921.5080, found: 921.5083 .

\section{3-O-descladinosyl-3-O-(3-pyridyl)acetyl-6-O-} methyl- I I, I 2-dideoxy- I 2, I I-(oxycarbonyI[(3[5-(pyridin-3-yl)-2H-tetrazol-2-yl])propyl]imino) erythromycin A (compound 10c)

Yield $55.8 \%$. melting point $(\mathrm{Mp})=173^{\circ} \mathrm{C}-175^{\circ} \mathrm{C}$; ${ }^{1} \mathrm{H}$ NMR $\left(300 \mathrm{MHz}, \mathrm{CDCl}_{3}\right) \delta 9.33(\mathrm{~s}, 1 \mathrm{H}), 8.57$ (d, J=40.9 Hz, 3H), 8.39 (d, J=7.9 Hz, 1H), 7.69 (d, J=7.7 Hz, 1H), 7.43-7.32 $(\mathrm{m}, 1 \mathrm{H}), 7.26(\mathrm{~s}, 1 \mathrm{H}), 4.98(\mathrm{t}, \mathrm{J}=11.6 \mathrm{~Hz}, 2 \mathrm{H}), 4.76(\mathrm{dd}$, $\mathrm{J}=10.1,6.8 \mathrm{~Hz}, 2 \mathrm{H}), 3.82(\mathrm{~d}, \mathrm{~J}=7.0 \mathrm{~Hz}, 1 \mathrm{H}), 3.67(\mathrm{~d}, \mathrm{~J}=4.0 \mathrm{~Hz}$, $4 \mathrm{H}), 3.13-2.98(\mathrm{~m}, 3 \mathrm{H}), 2.91(\mathrm{~s}, 3 \mathrm{H}), 2.85$ (dd, J=11.1, 6.8 $\mathrm{Hz}, 1 \mathrm{H}), 2.49-2.36(\mathrm{~m}, 3 \mathrm{H}), 2.23(\mathrm{~s}, 6 \mathrm{H}), 2.13-2.05(\mathrm{~m}, 2 \mathrm{H})$, 1.90 (dd, J=13.6, $6.9 \mathrm{~Hz}, 1 \mathrm{H}), 1.67-1.60(\mathrm{~m}, 1 \mathrm{H}), 1.58-1.46$ $(\mathrm{m}, 4 \mathrm{H}), 1.38(\mathrm{~s}, 3 \mathrm{H}), 1.20(\mathrm{~d}, \mathrm{~J}=4.7 \mathrm{~Hz}, 5 \mathrm{H}), 1.09$ (dd, J=12.4, $6.6 \mathrm{~Hz}, 10 \mathrm{H}), 0.98(\mathrm{~d}, \mathrm{~J}=6.7 \mathrm{~Hz}, 3 \mathrm{H}), 0.88$ (d, J=6.6 Hz, $3 \mathrm{H}), 0.79(\mathrm{t}, \mathrm{J}=7.2 \mathrm{~Hz}, 3 \mathrm{H})$. HRMS (ESI) $\mathrm{m} / \mathrm{z}$ calcd for $\mathrm{C}_{47} \mathrm{H}_{68} \mathrm{~N}_{8} \mathrm{O}_{11}[\mathrm{M}+\mathrm{H}]^{+}:$921.5080, found: 921.5082 .

\section{3-O-descladinosyl-3-O-(3-pyridyl)acetyl-6-O-} methyl- I I, I 2-dideoxy- I 2, I I-(oxycarbonyl[(3[5-(pyridin-4-yl)-2H-tetrazol-2-yl])propyl]imino) erythromycin $\mathrm{A}$ (compound I0d)

Yield $47.9 \%$. Mp $=170^{\circ} \mathrm{C}-172{ }^{\circ} \mathrm{C}$; ${ }^{1} \mathrm{H}$ NMR $(300 \mathrm{MHz}$, $\left.\mathrm{CDCl}_{3}\right) \delta 9.34(\mathrm{~s}, 1 \mathrm{H}), 8.66(\mathrm{~s}, 1 \mathrm{H}), 8.52(\mathrm{~s}, 2 \mathrm{H}), 8.40$ (d, J=7.9 Hz, 1H), 7.70 (d, J=7.9 Hz, 1H), 7.38 (dd, J=7.8, $4.8 \mathrm{~Hz}, 1 \mathrm{H}), 7.29$ (s, 1H), 4.99 (t, J=11.3 Hz, 2H), $4.84-4.68$ (m, 2H), 3.83 (d, J=7.0 Hz, 2H), 3.68 (d, J=3.9 Hz, 3H), 3.12-3.03 (m, 2H), $2.92(\mathrm{~s}, 3 \mathrm{H}), 2.88-2.82(\mathrm{~m}, 1 \mathrm{H}), 2.47-2.38$ (m, 2H), 2.25 (s, 6H), $2.13(\mathrm{~s}, 2 \mathrm{H}), 1.95-1.86(\mathrm{~m}, 1 \mathrm{H}), 1.56$ (d, J=7.0 Hz, 2H), 1.39 (s, 3H), 1.20 (s, 3H), 1.14-1.09 (m, 6H), $1.06(\mathrm{~s}, 1 \mathrm{H}), 0.99(\mathrm{~d}, \mathrm{~J}=6.7 \mathrm{~Hz}, 3 \mathrm{H}), 0.89(\mathrm{~d}, \mathrm{~J}=6.6 \mathrm{~Hz}, 3 \mathrm{H}), 0.80$ $(\mathrm{t}, \mathrm{J}=7.2 \mathrm{~Hz}, 3 \mathrm{H}){ }^{13} \mathrm{C} \mathrm{NMR}\left(75 \mathrm{MHz}, \mathrm{CDCl}_{3}\right) \delta 215.68,174.25$, $170.43,162.77,157.32,151.00,150.55,150.29,148.84,148.13$, 137.07, 134.22, 123.64,103.78, 82.92, 80.91, 78.42, 78.35, $70.28,69.63,65.96,60.56,51.14,50.06,45.54,42.93,41.32$, $40.29,38.76,38.45,38.38,36.37,30.92,28.17,27.13,22.01$, 19.44, 18.78, 21.05, 14.22, 14.16, 10.20, 8.85. HRMS (ESI) m/z calcd for $\mathrm{C}_{47} \mathrm{H}_{68} \mathrm{~N}_{8} \mathrm{O}_{11}[\mathrm{M}+\mathrm{H}]^{+}$: 921.5080 , found: 921.5083 .

3-O-descladinosyl-3-O-(3-pyridyl)acetyl-6-Omethyl- I I, I 2-dideoxy- I 2, I I-(oxycarbonyI[(4[5-(pyridin-2-yl)-IH-tetrazol- I-yl])butyl]imino) erythromycin A (compound I0e)

Yield of 52.3\%. ${ }^{13} \mathrm{C} \mathrm{NMR}\left(75 \mathrm{MHz}, \mathrm{CDCl}_{3}\right) \delta 215.74,182.80$, $176.89,174.13,170.75,157.31,151.58,149.73,148.14$, $144.82,137.83,137.32,129.84,125.24,124.39,123.69$, 
$103.32,82.75,81.21,78.34,76.47,69.65,68.41,65.82$, $60.29,49.89,49.18,45.50,44.93,42.88,39.47,38.66,38.14$, $36.37,29.37,27.52,22.65,20.80,19.48,18.73,15.05,14.12$, $10.15,8.86,8.41$. HRMS (ESI) $\mathrm{m} / \mathrm{z}$ calcd for $\mathrm{C}_{48} \mathrm{H}_{70} \mathrm{~N}_{8} \mathrm{O}_{11}$ $[\mathrm{M}+\mathrm{H}]^{+}$: 935.5237 , found: 935.5232 .

\section{3-O-descladinosyl-3-O-(3-pyridyl)acetyl-6-O-} methyl- I I, I 2-dideoxy- I 2, I I-(oxycarbony|[(4[5-(pyridin-2-yl)-2H-tetrazol-2-yl])butyl]imino) erythromycin $\mathrm{A}$ (compound IOf)

Yield 55.4\%. ${ }^{1} \mathrm{H}$ NMR (300 MHz, $\left.\mathrm{CDCl}_{3}\right) \delta 8.72$ (d, J=4.0 Hz, $1 \mathrm{H}), 8.57$ (s, 1H), 8.51 (d, J=3.6 Hz, 1H), 8.18 (d, J=7.9 Hz, 1H), 7.79 (ddd, J=10.1, 5.9, 2.0 Hz, 2H), 7.39-7.28 (m, 2H), $6.27(\mathrm{~s}, 3 \mathrm{H}), 5.06-4.86(\mathrm{~m}, 2 \mathrm{H}), 4.73(\mathrm{t}, \mathrm{J}=7.1 \mathrm{~Hz}, 2 \mathrm{H}), 3.86$ (d, J=7.1 Hz, 1H), 3.75 (d, J=2.3 Hz, 1H), 3.70-3.62 (m, 3H), $3.28-3.15(\mathrm{~m}, 1 \mathrm{H}), 3.05(\mathrm{dd}, \mathrm{J}=14.9,7.3 \mathrm{~Hz}, 2 \mathrm{H}), 2.95(\mathrm{~s}, 2 \mathrm{H})$, 2.89-2.73 (m, 2H), 2.45 (s, 3H), 2.18-2.07 (m, 3H), 1.95-1.82 (m, 1H), 1.77-1.45 (m, 6H), 1.37 (s, 3H), 1.22 (d, J=5.7 Hz, $3 \mathrm{H}), 1.16$ (s, 9H), 1.08 (t, J=6.8 Hz, 6H), 0.98 (d, J=6.7 Hz, $3 \mathrm{H}), 0.86(\mathrm{~d}, \mathrm{~J}=6.8 \mathrm{~Hz}, 3 \mathrm{H}), 0.77$ (t, J=7.3 Hz, 3H). ${ }^{13} \mathrm{C} \mathrm{NMR}$ $\left(75 \mathrm{MHz}, \mathrm{CDCl}_{3}\right) \delta 215.79,183.82,174.10,170.63,164.60$, $157.42,150.17,149.74,148.07,146.84,137.96,137.09$, $124.71,123.74,122.42,103.47,82.80,81.38,78.48,78.42$, $69.87,68.62,65.81,60.36,53.01,50.08,45.53,42.89,39.61$, $38.72,38.07,36.37,29.66,27.35,26.93,24.13,21.98,20.87$, 19.53, 18.81, 15.02, 14.21, 10.22, 8.93. HRMS (ESI) m/z calcd for $\mathrm{C}_{48} \mathrm{H}_{70} \mathrm{~N}_{8} \mathrm{O}_{11}[\mathrm{M}+\mathrm{H}]^{+}$: 935.5237 , found: 935.5231 .

\section{3-O-descladinosyl-3-O-(3-pyridyl)acetyl-6-O- methyl- I I, I 2-dideoxy- I 2, I I-(oxycarbony|[(4- [5-(pyridin-3-yl)-2H-tetrazol-2-yl]butyl)imino]) erythromycin A (compound IOg)}

Yield 51.2\%. Mp $=133^{\circ} \mathrm{C}-135^{\circ} \mathrm{C}$; ${ }^{1} \mathrm{H}$ NMR $(300 \mathrm{MHz}$, $\left.\mathrm{CDCl}_{3}\right) \delta 9.34(\mathrm{~d}, \mathrm{~J}=1.5 \mathrm{~Hz}, 1 \mathrm{H}), 8.67$ (dd, J=4.9, $1.6 \mathrm{~Hz}$, $1 \mathrm{H}), 8.62-8.49(\mathrm{~m}, 2 \mathrm{H}), 8.41(\mathrm{dt}, \mathrm{J}=8.0,1.9 \mathrm{~Hz}, 1 \mathrm{H})$, $7.76(\mathrm{~d}, \mathrm{~J}=7.9 \mathrm{~Hz}, 1 \mathrm{H}), 7.40(\mathrm{dd}, \mathrm{J}=8.0,4.8 \mathrm{~Hz}, 1 \mathrm{H})$, $7.31(\mathrm{dd}, \mathrm{J}=7.7,4.8 \mathrm{~Hz}, 1 \mathrm{H}), 4.97$ (dd, J=10.0, $3.7 \mathrm{~Hz}$, $2 \mathrm{H}), 4.75$ (dd, J=14.0, $6.3 \mathrm{~Hz}, 3 \mathrm{H}), 3.85$ (d, J=7.2 Hz, $1 \mathrm{H}), 3.80-3.65(\mathrm{~m}, 6 \mathrm{H}), 3.48(\mathrm{~s}, 3 \mathrm{H}), 3.19$ (dd, J=9.9, $7.3 \mathrm{~Hz}, 1 \mathrm{H}), 3.06$ (d, J=6.8 Hz, 2H), 2.99 (s, 3H), 2.87 (dd, J=11.1, 6.9 Hz, 1H), 2.65-2.41 (m, 2H), 2.37 (s, $6 \mathrm{H}), 2.12(\mathrm{dd}, \mathrm{J}=14.7,7.3 \mathrm{~Hz}, 3 \mathrm{H}), 1.88$ (s, 4H), 1.73 (d, J=7.6 Hz, 2H), 1.65-1.55 (m, 3H), $1.40(\mathrm{~s}, 3 \mathrm{H}), 1.26$ (d, J=6.3 Hz, 3H), 1.14 (d, J=2.3 Hz, 3H), 1.09 (s, 3H), $1.01(\mathrm{~d}, \mathrm{~J}=6.8 \mathrm{~Hz}, 3 \mathrm{H}), 0.87(\mathrm{~d}, \mathrm{~J}=6.8 \mathrm{~Hz}, 3 \mathrm{H}), 0.81(\mathrm{t}$, $\mathrm{J}=7.3 \mathrm{~Hz}, 3 \mathrm{H}) .{ }^{13} \mathrm{C} \mathrm{NMR}\left(75 \mathrm{MHz}, \mathrm{CDCl}_{3}\right) \delta 215.82$, $174.13,170.46,162.60,157.40,150.92,150.25,148.72$, $147.96,137.22,134.15,129.40,123.83,123.68,123.54$,
$103.57,82.77,80.86,78.38,78.33,76.57,70.16,69.36$, $65.90,60.29,52.91,50.04,45.56,42.77,40.21,38.75$, $38.31,36.30,28.47,26.98,24.14,21.95,20.99,19.53$, $18.79,14.93,14.20,10.23,8.86$. HRMS (ESI) m/z calcd for $\mathrm{C}_{48} \mathrm{H}_{70} \mathrm{~N}_{8} \mathrm{O}_{11}[\mathrm{M}+\mathrm{H}]^{+}$: 935.5237, found: 935.5234 .

3-O-descladinosyl-3-O-(3-pyridyl)acetyl-6-Omethyl- I I, I2-dideoxy- I 2, I I-(oxycarbony|[(4[5-(pyridin-4-yl)-2H-tetrazol-2-yl])butyl]imino) erythromycin $\mathrm{A}$ (compound IOh)

Yield 47.9\%. ${ }^{1} \mathrm{H}$ NMR $\left(300 \mathrm{MHz}, \mathrm{CDCl}_{3}\right) \delta 8.68$ (d, J=5.5 Hz, 2H), 8.60-8.37 (m, 2H), 7.97 (d, J=6.0 Hz, 2H), $7.73(\mathrm{~d}, \mathrm{~J}=7.9 \mathrm{~Hz}, 1 \mathrm{H}), 7.36-7.21(\mathrm{~m}, 1 \mathrm{H}), 4.91$ (d, J=11.0 Hz, 2H), 4.70 (t, J=7.1 Hz, 2H), 3.83 (d, J=7.1 $\mathrm{Hz}, 1 \mathrm{H}), 3.77-3.57$ (m, 6H), 3.15 (dd, J=10.0, $7.3 \mathrm{~Hz}, 1 \mathrm{H}$ ), 3.06-3.00 (m, 1H), $2.93(\mathrm{~d}, \mathrm{~J}=4.3 \mathrm{~Hz}, 3 \mathrm{H}), 2.82(\mathrm{dd}, \mathrm{J}=11.1$, $6.8 \mathrm{~Hz}, 1 \mathrm{H}), 2.55-2.39(\mathrm{~m}, 2 \mathrm{H}), 2.29(\mathrm{~s}, 3 \mathrm{H}), 2.10(\mathrm{~s}, 3 \mathrm{H})$, $2.05(\mathrm{dd}, \mathrm{J}=9.0,5.7 \mathrm{~Hz}, 3 \mathrm{H}), 1.94-1.78(\mathrm{~m}, 1 \mathrm{H}), 1.70(\mathrm{dd}$, $\mathrm{J}=15.6,7.7 \mathrm{~Hz}, 2 \mathrm{H}), 1.58$ (d, J=14.9 Hz, 3H), $1.36(\mathrm{~s}, 3 \mathrm{H})$, $1.22(\mathrm{~s}, 3 \mathrm{H}), 1.19(\mathrm{~s}, 1 \mathrm{H}), 1.14(\mathrm{~s}, 6 \mathrm{H}), 1.07$ (dd, J=8.3, 5.4 $\mathrm{Hz}, 6 \mathrm{H}), 0.96(\mathrm{~d}, \mathrm{~J}=6.7 \mathrm{~Hz}, 3 \mathrm{H}), 0.81-0.78(\mathrm{~m}, 3 \mathrm{H}), 0.73$ $(\mathrm{d}, \mathrm{J}=7.2 \mathrm{~Hz}, 3 \mathrm{H}) .{ }^{13} \mathrm{C} \mathrm{NMR}\left(75 \mathrm{MHz}, \mathrm{CDCl}_{3}\right) \delta 215.84$, 174.12, 170.51, 162.78, 157.37, 150.11, 148.44, 137.37, $135.13,129.53,123.59,120.87,103.66,82.72,80.96,78.3$, $76.48,70.08,69.14,65.74,60.23,53.01,49.99,45.54,44.81$, $42.72,39.86,38.52,38.18,36.31,30.90,30.10,29.62,28.51$, 27.52, 26.93, 24.10, 22.62, 21.91, 20.96, 19.50, 18.76, 14.87, 14.16, 10.21, 8.77. HRMS (ESI) $\mathrm{m} / \mathrm{z}$ calcd for $\mathrm{C}_{48} \mathrm{H}_{70} \mathrm{~N}_{8} \mathrm{O}_{11}$ $[\mathrm{M}+\mathrm{H}]^{+}$: 935.5237, found: 935.5236 .

3-O-descladinosyl-3-O-(3-pyridyl)acetyl-6-Omethyl- I I, I 2-dideoxy- I 2, I I-(oxycarbony|[(5[5-(pyridin-2-yl)-IH-tetrazol- |-yl])pentyl]imino) erythromycin $\mathrm{A}$ (compound $\mathrm{IO}$ )

Yield 47.8\%. ${ }^{1} \mathrm{H}$ NMR (300 $\left.\mathrm{MHz}, \mathrm{CDCl}_{3}\right) \delta 8.70$ (d, J=4.8 Hz, 1H), 8.55-8.45 (m, 2H), 8.28 (d, J=7.9 Hz, 1H), 7.84 (td, J=7.8, $1.7 \mathrm{~Hz}, 1 \mathrm{H}), 7.69(\mathrm{~d}, \mathrm{~J}=7.9 \mathrm{~Hz}, 1 \mathrm{H})$, 7.38 (ddd, J=7.6, 4.9, $1.0 \mathrm{~Hz}, 1 \mathrm{H}), 7.25$ (dd, J=8.0, $4.7 \mathrm{~Hz}$, $1 \mathrm{H}), 4.99-4.88(\mathrm{~m}, 4 \mathrm{H}), 3.83(\mathrm{~d}, \mathrm{~J}=7.2 \mathrm{~Hz}, 1 \mathrm{H}), 3.69$ (d, J=2.4 Hz, 3H), 3.63 (s, 1H), 3.59-3.50 (m, 2H), 3.07 (ddd, J=14.4, 8.7, 4.6 Hz, 3H), 2.93 (s, 3H), 2.84 (dd, J=11.1, $6.7 \mathrm{~Hz}, 1 \mathrm{H}), 2.24(\mathrm{~s}, 6 \mathrm{H}), 2.12(\mathrm{~s}, 6 \mathrm{H}), 2.03-1.91(\mathrm{~m}, 2 \mathrm{H})$, $1.64(\mathrm{~d}, \mathrm{~J}=9.2 \mathrm{~Hz}, 2 \mathrm{H}), 1.55$ (dd, J=12.9, $4.4 \mathrm{~Hz}, 3 \mathrm{H}), 1.35$ $(\mathrm{d}, \mathrm{J}=4.0 \mathrm{~Hz}, 3 \mathrm{H}), 1.23(\mathrm{~s}, 3 \mathrm{H}), 1.20-1.13(\mathrm{~m}, 3 \mathrm{H}), 1.13-1.08$ (m, 6H), 1.06 (d, J=7.1 Hz, 3H), 0.96 (d, J=6.8 Hz, 3H), $0.86(\mathrm{~d}, \mathrm{~J}=6.7 \mathrm{~Hz}, 3 \mathrm{H}), 0.74(\mathrm{t}, \mathrm{J}=7.3 \mathrm{~Hz}, 3 \mathrm{H}) .{ }^{13} \mathrm{C}$ NMR $\left(75 \mathrm{MHz}, \mathrm{CDCl}_{3}\right) \delta 215.73,207.12,174.08,170.47,157.29$, $151.62,150.26,149.60,148.78,144.89,137.36,137.13$, 
$129.33,125.24,124.45,123.52,103.71,82.62,80.94,78.39$, $76.55,70.25,69.57,65.91,60.37,50.00,49.61,21.99$, $21.05,19.55,18.82,15.00,14.25,14.19,10.24,8.87,8.83$. HRMS (ESI) $\mathrm{m} / \mathrm{z}$ calcd for $\mathrm{C}_{49} \mathrm{H}_{72} \mathrm{~N}_{8} \mathrm{O}_{11}[\mathrm{M}+\mathrm{H}]^{+}$: 949.5393 , found: 949.5395 .

\section{3-O-descladinosyl-3-O-(3-pyridyl)acetyl-6-O-} methyl- I I, I 2-dideoxy- I 2, I I-(oxycarbony|[(5[5-(pyridin-2-yl)-2H-tetrazol-2-yl]pentyl)imino]) erythromycin $A$ (compound $10 \mathrm{j}$ )

Yield 62.7\%. ${ }^{1} \mathrm{H} \mathrm{NMR}\left(300 \mathrm{MHz}, \mathrm{CDCl}_{3}\right) \delta 8.76(\mathrm{~d}, \mathrm{~J}=4.1 \mathrm{~Hz}$, $1 \mathrm{H}), 8.58(\mathrm{~s}, 1 \mathrm{H}), 8.56-8.47(\mathrm{~m}, 1 \mathrm{H}), 8.22(\mathrm{~d}, \mathrm{~J}=7.9 \mathrm{~Hz}, 1 \mathrm{H})$, 7.90-7.74 (m, 2H), 7.41-7.28 (m, 2H), 4.99 (d, J=11.1 Hz, $2 \mathrm{H}), 4.68(\mathrm{t}, \mathrm{J}=7.2 \mathrm{~Hz}, 2 \mathrm{H}), 3.88(\mathrm{~d}, \mathrm{~J}=7.1 \mathrm{~Hz}, 1 \mathrm{H}), 3.80-3.69$ $(\mathrm{m}, 3 \mathrm{H}), 3.66(\mathrm{~s}, 1 \mathrm{H}), 3.65-3.52(\mathrm{~m}, 2 \mathrm{H}), 3.25(\mathrm{dd}, \mathrm{J}=10.0,7.2$ $\mathrm{Hz}, 1 \mathrm{H}), 3.02$ (dd, J=14.1, $6.8 \mathrm{~Hz}, 6 \mathrm{H}), 2.91-2.81(\mathrm{~m}, 1 \mathrm{H})$, 2.77-2.64 (m, 1H), 2.58-2.48 (m, 1H), $2.42(\mathrm{~s}, 3 \mathrm{H}), 2.21$ $(\mathrm{t}, \mathrm{J}=5.9 \mathrm{~Hz}, 3 \mathrm{H}), 2.17-2.11(\mathrm{~m}, 3 \mathrm{H}), 1.70(\mathrm{dd}, \mathrm{J}=18.7,11.9 \mathrm{~Hz}$, $3 \mathrm{H}), 1.39$ (s, 3H), 1.26 (d, J=1.7 Hz, 3H), 1.24 (s, 3H), 1.21 (s, 3H), 1.18-1.17 (m, 3H), 1.12-1.07 (m, 6H), 0.99 (d, J=6.8 $\mathrm{Hz}, 3 \mathrm{H}), 0.92$ (d, J=6.7 Hz, 3H), 0.79 (t, J=7.3 Hz, 3H). ${ }^{13} \mathrm{C}$ NMR (75 MHz, $\left.\mathrm{CDCl}_{3}\right) \delta 215.90,184.13,174.19,170.71$, $164.70,157.45,150.30,149.83,148.17,146.96,138.02$, $137.19,129.95,128.38,127.94,125.88,124.81,123.81$, $122.51,103.66,82.79,81.49,78.64,78.55,77.37,70.08$, $68.85,65.85,60.52,53.49,50.18,45.65,45.06,43.43,43.02$, 40.73-40.56 (m), 39.71, 38.72, 38.51, 38.19, 36.45, 31.99, $31.50,30.25,29.77,29.09,27.55,26.42-26.15$ (m), 23.96, 22.76, 22.11, 20.99, 19.64, 18.93, 15.14, 14.49-14.07 (m), $10.35,9.03,8.63$. HRMS (ESI) $\mathrm{m} / \mathrm{z}$ calcd for $\mathrm{C}_{49} \mathrm{H}_{72} \mathrm{~N}_{8} \mathrm{O}_{11}$ $[\mathrm{M}+\mathrm{H}]^{+}:$949.5393, found: 949.5389 .

\section{3-O-descladinosyl-3-O-(3-pyridyl)acetyl-6-O-} methyl- I I, I 2-dideoxy- I 2, I I-(oxycarbony|[(5[5-(pyridin-3-yl)-2H-tetrazol-2-yl]pentyl)imino]) erythromycin A (compound I0k)

Yield 56.3\%. $\mathrm{Mp}=139^{\circ} \mathrm{C}-141^{\circ} \mathrm{C}$; ${ }^{1} \mathrm{H}$ NMR $(300 \mathrm{MHz}$, $\left.\mathrm{CDCl}_{3}\right) \delta 9.34(\mathrm{~s}, 1 \mathrm{H}), 8.63(\mathrm{~d}, \mathrm{~J}=33.2 \mathrm{~Hz}, 2 \mathrm{H}), 8.55-8.34$ (m, 2H), 7.77 (d, J=7.8 Hz, 2H), 7.45-7.40 (m, 1H), 7.37-7.27 (m, 1H), 5.05 (d, J=37.1 Hz, 3H), 4.97 (d, J=11.0 Hz, 2H), 4.65 (t, J=7.0 Hz, 2H), 3.88 (d, J=7.0 Hz, 1H), 3.84-3.69 (m, 3H), 3.65-3.54 (m, 3H), 3.39-3.20 (m, 1H), 3.07 (dd, $\mathrm{J}=14.5,7.2 \mathrm{~Hz}, 6 \mathrm{H}), 2.97$ (s, 3H), 2.85 (d, J=11.0 Hz, 2H), 2.49 (s, 6H), 2.26-2.03 (m, 3H), 1.88 (dd, J=13.4, 7.2 Hz, $1 \mathrm{H}), 1.68(\mathrm{~s}, 3 \mathrm{H}), 1.57(\mathrm{~d}, \mathrm{~J}=8.3 \mathrm{~Hz}, 2 \mathrm{H}), 1.38-1.32(\mathrm{~m}, 6 \mathrm{H})$, $1.23(\mathrm{~s}, 3 \mathrm{H}), 1.17(\mathrm{~s}, 6 \mathrm{H}), 1.10(\mathrm{~s}, 3 \mathrm{H}), 0.98(\mathrm{~d}, \mathrm{~J}=6.4 \mathrm{~Hz}$, $3 \mathrm{H}), 0.91$ (d, J=6.4 Hz, 3H), 0.77 (s, 3H). ${ }^{13} \mathrm{C}$ NMR $(75 \mathrm{MHz}$, $\left.\mathrm{CDCl}_{3}\right) \delta 214.29,183.33,173.95,170.73,162.55,157.35$,
$150.69,149.77,148.13,147.71,137.95,134.47,123.84$, $103.36,82.70,78.41,69.87,68.60,65.91,60.39,53.33$, $50.08,45.61,42.91,39.78,38.72,38.21,29.32,28.97,27.33$, 26.43, 23.82, 20.87, 19.56, 18.84, 15.08, 14.25, 10.27, 8.95, 8.61. HRMS (ESI) $\mathrm{m} / \mathrm{z}$ calcd for $\mathrm{C}_{49} \mathrm{H}_{72} \mathrm{~N}_{8} \mathrm{O}_{11}[\mathrm{M}+\mathrm{H}]^{+}$: 949.5393, found: 949.5392 .

\section{3-O-descladinosyl-3-O-(3-pyridyl)acetyl-6-O- methyl- I I, I 2-dideoxy- I 2, I I-(oxycarbony|[(5- [5-(pyridin-4-yl)-2H-tetrazol-2-yl])pentyl]imino) erythromycin $\mathrm{A}$ (compound I0I)}

Yield 54.2\%. ${ }^{1} \mathrm{H}$ NMR (300 MHz, $\mathrm{CDCl}_{3}$ ) $\delta 8.71$ (dd, J=4.6, $1.5 \mathrm{~Hz}, 2 \mathrm{H}$ ), 8.54 (d, J=1.7 Hz, 1H), 8.49 (dd, J=4.8, $1.4 \mathrm{~Hz}$, 1H), 7.99 (dd, J=4.6, 1.5 Hz, 2H), 7.74 (d, J=7.9 Hz, 1H), 7.28 (d, J=2.9 Hz, 1H), 4.95 (d, J=11.1 Hz, 2H), 4.64 (t, J=7.1 Hz, 2H), 3.85 (d, J=7.1 Hz, 1H), 3.73 (s, 2H), 3.66 (dd, J=11.6, $7.4 \mathrm{~Hz}, 2 \mathrm{H}), 3.56$ (dd, J=9.2, $6.4 \mathrm{~Hz}, 2 \mathrm{H}), 3.22$ (dd, J=10.1, $7.2 \mathrm{~Hz}, 1 \mathrm{H}$ ), 3.01 (q, J=7.3 Hz, 5H), 2.95 (s, 3H), 2.39 (s, 3H), 2.15-2.03 (m, 3H), 1.92-1.80 (m, 1H), $1.60(\mathrm{~s}, 3 \mathrm{H}), 1.51$ (d, J=12.7 Hz, 3H), 1.35 (s, 3H), 1.26 (d, J=7.3 Hz, 3H), $1.21(\mathrm{~d}, \mathrm{~J}=4.0 \mathrm{~Hz}, 3 \mathrm{H}), 1.14$ (s, 6H), 1.09-1.04 (m, 6H), 0.95 (d, J=6.8 Hz, 3H), 0.87 (d, J=6.7 Hz, 3H), 0.74 (t, J=7.3 Hz, $3 \mathrm{H}) .{ }^{13} \mathrm{C} \mathrm{NMR}\left(75 \mathrm{MHz}, \mathrm{CDCl}_{3}\right.$ ) $\delta 215.91,183.67,174.21$, $170.72,162.77,157.34,150.07,149.79,148.17,137.82$, $135.27,129.76,123.70,120.98,103.53,82.70,81.21,78.37$, $69.92,68.75,65.76,60.35,53.40,50.04,45.56,45.17,43.26$, $42.87,39.65,38.72,38.22,36.35,28.95,27.48,26.38,23.75$, $21.98,20.90,19.53,18.82,15.03,14.21,10.25,8.92,8.56$. HRMS (ESI) $\mathrm{m} / \mathrm{z}$ calcd for $\mathrm{C}_{49} \mathrm{H}_{72} \mathrm{~N}_{8} \mathrm{O}_{11}[\mathrm{M}+\mathrm{H}]^{+}:$: 949.5393, found: 949.5392 .

3-O-descladinosyl-3-O-(3-pyridyl)acetyl-6-O-methylI I, I 2-dideoxy- I 2, I I-(oxycarbony|[(4-[5-phenyl-2Htetrazol-2-yl])pentyl]imino)erythromycin A (compound $10 \mathrm{~m}$ )

Yield 56.4\%. ${ }^{1} \mathrm{H}$ NMR (300 MHz, $\left.\mathrm{CDCl}_{3}\right) \delta 8.60-8.47$ (m, 2H), 8.18-8.08 (m, 2H), 7.75 (d, J=8.0 Hz, 1H), 7.46 $(\mathrm{d}, \mathrm{J}=6.6 \mathrm{~Hz}, 3 \mathrm{H}), 7.30$ (dd, J=7.8, 4.9 Hz, 1H), 5.01 (d, J=11.0 $\mathrm{Hz}, 2 \mathrm{H}), 4.65$ (t, J=7.2 Hz, 2H), 3.98-3.81 (m, 2H), 3.74 (s, 3H), 3.66-3.54 (m, 2H), 3.26 (ddd, J=24.7, 16.2, 7.2 Hz, $3 \mathrm{H}), 3.01(\mathrm{~s}, 3 \mathrm{H}), 2.91-2.84(\mathrm{~m}, 3 \mathrm{H}), 2.60-2.45(\mathrm{~m}, 2 \mathrm{H})$, $2.37(\mathrm{~s}, 3 \mathrm{H}), 2.26(\mathrm{~d}, \mathrm{~J}=5.0 \mathrm{~Hz}, 1 \mathrm{H}), 2.18-2.10(\mathrm{~m}, 3 \mathrm{H})$, $1.96-1.78(\mathrm{~m}, 2 \mathrm{H}), 1.72(\mathrm{~d}, \mathrm{~J}=7.1 \mathrm{~Hz}, 2 \mathrm{H}), 1.67$ (s, 1H), 1.60 (d, J=10.3 Hz, 2H), 1.46 (s, 1H), 1.40 (s, 3H), 1.27 $(\mathrm{d}, \mathrm{J}=2.7 \mathrm{~Hz}, 3 \mathrm{H}), 1.20$ (s, 3H), $1.18(\mathrm{~s}, 3 \mathrm{H}), 1.15$ (d, J=2.8 Hz, $3 \mathrm{H}), 1.09$ (s, 3H), 1.01 (d, J=6.7 Hz, 3H), 0.93 (d, J=6.8 Hz, $3 \mathrm{H}), 0.80$ (t, J=7.2 Hz, 3H). ${ }^{13} \mathrm{C} \mathrm{NMR}\left(75 \mathrm{MHz}, \mathrm{CDCl}_{3}\right) \delta$ $215.77,174.13,170.56,166.98,157.33,150.23,148.72$, 
$137.35,130.14,129.49,126.85,123.61,102.62,82.67$, $81.20,78.36,70.12,69.04,66.06,60.42,53.05,50.11,45.60$, $43.37,40.21,38.82,36.32,31.44,30.17,29.04,27.52,26.50$, 23.88, 22.03, 21.01, 19.58, 18.86, 15.08, 14.30, 10.28, 8.95. HRMS (ESI) $\mathrm{m} / \mathrm{z}$ calcd for $\mathrm{C}_{50} \mathrm{H}_{73} \mathrm{~N}_{7} \mathrm{O}_{11}[\mathrm{M}+\mathrm{H}]^{+}:$948.5441, found: 948.5444 .

\section{3-O-descladinosyl-3-O-(3-pyridyl)acetyl-6-O-} methyl-I I, I 2-dideoxy- I 2, I I-(oxycarbony|[(3-[5(thiophen-2-yl)-2H-tetrazol-2-yl])pentyl]imino) erythromycin A (compound IOn)

Yield $60.6 \%$. Mp $=99^{\circ} \mathrm{C}-101^{\circ} \mathrm{C} ;{ }^{1} \mathrm{H}$ NMR $(300 \mathrm{MHz}$, $\left.\mathrm{CDCl}_{3}\right) \delta 8.51(\mathrm{dd}, \mathrm{J}=4.9,1.4 \mathrm{~Hz}, 2 \mathrm{H}), 7.76(\mathrm{dd}, \mathrm{J}=3.6$, $1.2 \mathrm{~Hz}, 1 \mathrm{H}), 7.70(\mathrm{dt}, \mathrm{J}=7.8,1.8 \mathrm{~Hz}, 1 \mathrm{H}), 7.40$ (dd, J=5.0, $1.2 \mathrm{~Hz}, 1 \mathrm{H}), 7.27$ (dd, J=7.6, $4.7 \mathrm{~Hz}, 1 \mathrm{H}), 7.10$ (dd, J=5.0, $3.7 \mathrm{~Hz}, 1 \mathrm{H}), 5.06-4.91(\mathrm{~m}, 2 \mathrm{H}), 4.59$ (t, J=7.2 Hz, 2H), $3.85(\mathrm{~d}, \mathrm{~J}=7.2 \mathrm{~Hz}, 1 \mathrm{H}), 3.74-3.68(\mathrm{~m}, 3 \mathrm{H}), 3.66(\mathrm{~s}, 1 \mathrm{H})$, 3.58 (dd, J=8.4, $5.9 \mathrm{~Hz}, 2 \mathrm{H}), 3.19-3.01$ (m, 3H), 2.98 (d, $\mathrm{J}=6.2 \mathrm{~Hz}, 3 \mathrm{H}), 2.93-2.80(\mathrm{~m}, 2 \mathrm{H}), 2.68-2.59(\mathrm{~m}, 1 \mathrm{H}), 2.23$ (d, J=4.4 Hz, 6H), 2.16-2.05 (m, 3H), 1.78-1.66 (m, 2H), 1.57 (t, J=10.9 Hz, 3H), 1.38 (s, 3H), 1.26 (s, 3H), 1.16-1.04 (m, 12H), 0.98 (d, J=6.8 Hz, 3H), 0.90 (d, J=6.8 Hz, 3H), 0.80 (ddd, J=15.0, 5.9, $2.6 \mathrm{~Hz}, 6 \mathrm{H}) .{ }^{13} \mathrm{C}$ NMR $(75 \mathrm{MHz}$, $\left.\mathrm{CDCl}_{3}\right) \delta 215.75,174.10,170.43,161.04,157.32,150.27$, $148.80,137.11,129.27,127.76,123.52,103.79,82.67$, $81.01,78.43,70.32,69.66,65.92,60.42,53.12,50.09$, $45.84,45.59,43.34,42.94,40.29,38.6,36.28,28.97,28.11$, $26.45,23.82,22.02,21.07,19.56,18.84,15.01,14.24,10.26$, 8.89. HRMS (ESI) $\mathrm{m} / \mathrm{z}$ calcd for $\mathrm{C}_{48} \mathrm{H}_{71} \mathrm{~N}_{7} \mathrm{O}_{11} \mathrm{~S}[\mathrm{M}+\mathrm{H}]^{+}$: 954.5005, found: 954.5003 .

\section{3-O-descladinosyl-3-O-(3-pyridyl)acetyl-6-O- methyl- I I, I 2-dideoxy- I 2, I I-(oxycarbonyl[(3- [4-( I-methyl- I H-tetrazol-5-thio)]propyl]imino) erythromycin $\mathrm{A}$ (compound 100)}

Yield 53.2\%. Mp $=202^{\circ} \mathrm{C}-204^{\circ} \mathrm{C}$; ${ }^{1} \mathrm{H}$ NMR $(300 \mathrm{MHz}$, $\left.\mathrm{CDCl}_{3}\right) \delta 8.52(\mathrm{dd}, \mathrm{J}=4.9,1.4 \mathrm{~Hz}, 2 \mathrm{H}), 7.78-7.65(\mathrm{~m}, 1 \mathrm{H})$, $7.32-7.26(\mathrm{~m}, 1 \mathrm{H}), 4.96(\mathrm{dd}, \mathrm{J}=13.5,6.5 \mathrm{~Hz}, 2 \mathrm{H}), 3.90$ (s, 3H), 3.85 (d, J=7.2 Hz, 1H), 3.75 (t, J=7.2 Hz, 2H), 3.71 (d, J=1.9 Hz, 3H), 3.65 (s, 1H), 3.51-3.29 (m, 3H), 3.18-3.10 (m, 1H), $3.05(\mathrm{~d}, \mathrm{~J}=7.0 \mathrm{~Hz}, 2 \mathrm{H}), 2.99$ (s, 3H), 2.86 (dd, J=11.1, $6.7 \mathrm{~Hz}, 1 \mathrm{H}), 2.51(\mathrm{dd}, \mathrm{J}=9.1,5.4 \mathrm{~Hz}, 1 \mathrm{H}), 2.28(\mathrm{~s}, 6 \mathrm{H})$, 2.17-2.04 (m, 3H), 1.96-1.82 (m, 1H), 1.64-1.52 (m, 3H), 1.38 (s, 3H), 1.26 (dd, J=13.7, 10.6 Hz, 6H), 1.10 (dd, J=10.2, $6.6 \mathrm{~Hz}, 9 \mathrm{H}), 0.98$ (d, J=6.8 Hz, 3H), 0.90 (d, J=6.7 Hz, 3H), $0.79(\mathrm{t}, \mathrm{J}=7.3 \mathrm{~Hz}, 3 \mathrm{H}) .{ }^{13} \mathrm{C} \mathrm{NMR}\left(75 \mathrm{MHz}, \mathrm{CDCl}_{3}\right) \delta 215.71$, $174.17,170.51,157.37,154.17,150.31,148.86,137.13$, $129.31,123.55,103.73,82.83,80.94,78.40,76.58,70.24$,
$69.58,65.97,60.41,50.23,45.58,42.92,42.29,40.31,38.79$, $38.40,36.32,33.43,31.07,28.24,26.80,22.00,21.06,19.58$, $18.85,15.05,14.25,10.22,8.88$. HRMS (ESI) $\mathrm{m} / \mathrm{z}$ calcd for $\mathrm{C}_{43} \mathrm{H}_{67} \mathrm{~N}_{7} \mathrm{O}_{11} \mathrm{~S}[\mathrm{M}+\mathrm{H}]^{+}:$890.4692, found: 890.4691 .

3-O-descladinosyl-3-O-(3-pyridyl)acetyl-6-Omethyl- I I, I 2-dideoxy- I 2, I I-(oxycarbonyI[(4[4-( I-methyl-IH-tetrazol-5-thio)]butyl]imino) erythromycin A (compound I0p)

Yield 55.9\%. ${ }^{1} \mathrm{H}$ NMR (300 MHz, $\left.\mathrm{CDCl}_{3}\right) \delta 8.53(\mathrm{~d}, \mathrm{~J}=6.0 \mathrm{~Hz}$, 2H), 7.74 (d, J=7.9 Hz, 1H), 7.29 (dd, J=7.8, 4.9 Hz, 1H), 4.97 (t, J=10.2 Hz, 2H), 3.88 (s, 3H), 3.79-3.69 (m, 3H), $3.67-3.58(\mathrm{~m}, 3 \mathrm{H}), 3.47(\mathrm{~s}, 2 \mathrm{H}), 3.39(\mathrm{dd}, \mathrm{J}=8.2,5.2 \mathrm{~Hz}$, 2H), $3.15(\mathrm{dd}, \mathrm{J}=10.1,7.3 \mathrm{~Hz}, 1 \mathrm{H}), 3.04(\mathrm{~d}, \mathrm{~J}=6.6 \mathrm{~Hz}$, 2H), 2.96 (d, J=3.8 Hz, 3H), 2.91-2.77 (m, 1H), 2.59-2.48 (m, 1H), 2.46-2.33 (m, 1H), 2.29 (s, 5H), 2.23 (d, J=4.8 Hz, 2H), 2.19-2.14 (m, 2H), 2.09 (dd, J=12.2, 5.1 Hz, 2H), 1.81 (s, 3H), 1.59 (dd, J=18.7, $11.5 \mathrm{~Hz}, 3 \mathrm{H}), 1.38$ (s, 3H), 1.27 (s, 3H), 1.17 (d, J=3.9 Hz, 3H), 1.14 (s, 3H), 1.09 $(\mathrm{d}, \mathrm{J}=7.2 \mathrm{~Hz}, 3 \mathrm{H}), 0.99$ (d, J=6.7 Hz, 3H), $0.91(\mathrm{~d}, \mathrm{~J}=6.7 \mathrm{~Hz}$, $3 \mathrm{H}), 0.78$ (t, J=7.3 Hz, 3H). ${ }^{13} \mathrm{C}$ NMR $\left(75 \mathrm{MHz}, \mathrm{CDCl}_{3}\right)$ $\delta$ 215.76, 174.12,170.56, 157.32, 154.21, 150.26, 148.75, 137.26, 103.54, 82.70, 81.04, 78.46, 78.39, 77.49, 77.07, $76.64,70.15,69.29,66.04,60.45,50.00,45.58,42.96,40.25$, $38.80,38.43,38.34,36.31,33.33,32.87,28.66,26.72,26.10$, $22.03,21.00,19.57,18.81,15.06,14.27,14.21,10.26,8.91$ HRMS (ESI) $\mathrm{m} / \mathrm{z}$ calcd for $\mathrm{C}_{44} \mathrm{H}_{69} \mathrm{~N}_{7} \mathrm{O}_{11} \mathrm{~S}[\mathrm{M}+\mathrm{H}]^{+}: 904.4849$, found: 904.4844 .

3-O-descladinosyl-3-O-(3-pyridyl)acetyl-6-Omethyl-I I, I 2-dideoxy- I 2, I I-(oxycarbonyI[(5[4-(I-methyl-IH-tetrazol-5-thio)]penty|]imino) erythromycin $\mathrm{A}$ (compound $\mathrm{I0q}$ )

Yield $52.9 \% . \mathrm{Mp}=101^{\circ} \mathrm{C}-104^{\circ} \mathrm{C} ;{ }^{1} \mathrm{HNMR}\left(300 \mathrm{MHz}, \mathrm{CDCl}_{3}\right)$ $\delta$ 8.60-8.46 (m, 2H), $7.74(\mathrm{~d}, \mathrm{~J}=7.9 \mathrm{~Hz}, 1 \mathrm{H}), 7.35-7.27(\mathrm{~m}$, $1 \mathrm{H}), 4.98(\mathrm{~d}, \mathrm{~J}=11.0 \mathrm{~Hz}, 2 \mathrm{H}), 3.88(\mathrm{~s}, 3 \mathrm{H}), 3.74-3.67(\mathrm{~m}, 3 \mathrm{H})$, $3.65(\mathrm{~s}, 1 \mathrm{H}), 3.61-3.51(\mathrm{~m}, 2 \mathrm{H}), 3.33(\mathrm{t}, \mathrm{J}=7.1 \mathrm{~Hz}, 2 \mathrm{H}), 3.17$ (dd, J=10.0, $7.3 \mathrm{~Hz}, 1 \mathrm{H}), 3.08-3.02(\mathrm{~m}, 1 \mathrm{H}), 2.98(\mathrm{~s}, 2 \mathrm{H})$, 2.92-2.81 (m, 1H), 2.59-2.40 (m, 2H), 2.33 (s, 5H), 2.19$2.06(\mathrm{~m}, 2 \mathrm{H}), 1.84$ (dd, J=15.1, 7.6 Hz, 3H), 1.62 (d, J=11.9 $\mathrm{Hz}, 3 \mathrm{H}), 1.53-1.43$ (m, 3H), 1.38 (s, 3H), 1.26 (dd, J=13.3, $10.7 \mathrm{~Hz}, 6 \mathrm{H}), 1.17$ (s, 2H), 1.14-1.06 (m, 9H), 0.99 (d, J=6.8 $\mathrm{Hz}, 3 \mathrm{H}), 0.91(\mathrm{~d}, \mathrm{~J}=6.7 \mathrm{~Hz}, 3 \mathrm{H}), 0.78(\mathrm{t}, \mathrm{J}=7.3 \mathrm{~Hz}, 3 \mathrm{H}) .{ }^{13} \mathrm{C}$ NMR (75 MHz, $\left.\mathrm{CDCl}_{3}\right) \delta 215.77,174.11,170.57,157.33$, $154.38,150.17,148.65,137.34,129.48,123.60,103.63$, $82.65,81.09,78.50,78.39,70.19,69.31,65.94,60.42$, $50.07,45.58,43.40,42.94,40.14,38.81,38.42,38.32$, $36.30,33.33,33.28,28.76,27.48,26.52,25.81,22.04,21.00$, 


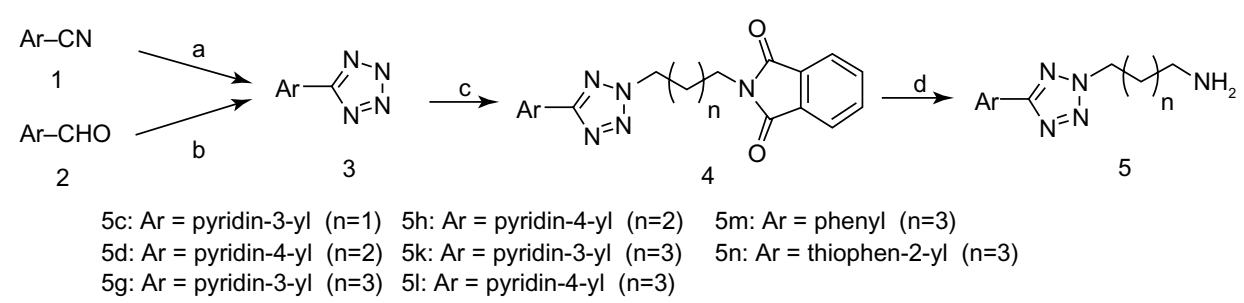

Figure I Synthesis of N2-substituted 5-aryl-tetrazolyl alkylamines.

Notes: Reagents and condition: (a) $\mathrm{NaN}_{3}, \mathrm{NH}_{4} \mathrm{Cl}$, DMF, $130^{\circ} \mathrm{C}$ and (b) i) $\mathrm{I}_{2}$, aq $\mathrm{NH}_{3}$, rt; ii) $\mathrm{NaN}_{3}$, ZnBr; reflux; (c) $\mathrm{N}$-(3-bromopropyl)phthalimide, $\mathrm{N}$-(4-bromobutyl) phthalimide or $\mathrm{N}$-(5-bromopentyl)phthalimide, $\mathrm{KCO}_{3}$, DMF, $80^{\circ} \mathrm{C}$ : (d) $\mathrm{NH}_{2} \mathrm{NH}_{2} \cdot \mathrm{HO}, \mathrm{EtOH} / \mathrm{CH}_{3} \mathrm{CN}$, reflux.

Abbreviations: Ar, aromatic nucleus; DMF, N, N-dimethyl formamide; aq, aqueous; rt, room temperature; EtOH, ethanol.

19.57, 18.85, 15.04, 14.27, 10.27, 8.91. HRMS (ESI) $\mathrm{m} / \mathrm{z}$ calcd for $\mathrm{C}_{45} \mathrm{H}_{71} \mathrm{~N}_{7} \mathrm{O}_{11} \mathrm{~S}[\mathrm{M}+\mathrm{H}]^{+}: 918.5005$, found: 918.4993.

\section{Minimum inhibitory concentration assay}

The standard antibiotics erythromycin A and clarithromycin were obtained from commercial sources. The antimicrobial susceptibility test in vitro against acylide derivatives and standard antibiotics was performed using broth microdilution method, according to National Committee for Clinical Laboratory Standards guidelines. Acylides and the reference drugs were dissolved in glacial acetic acid as mother liquor; the amount of acetic acid must be less than $2.5 \mu \mathrm{L} / \mathrm{mL}$ to exclude glacial acetic acid effect on antibacterial activity in vitro. Minimum inhibitory concentration $(\mu \mathrm{g} / \mathrm{mL})$ is used to report the antibacterial activities of the compounds in vitro.

All of the synthesized acylides and standard antibiotics were tested for in vitro antibacterial activity against two erythromycin-susceptible strains of $S$. aureus (American Type Culture Collection [ATCC] 25923, ATCC 6538), one strain of Staphylococcus epidermidis (ATCC 12228), two strains of Pseudomonas aeruginosa (ATCC 9027 and a clinical isolate of resistant PA 1317), and two strains of Escherichia coli (ATCC 8739, ATCC 25922).

\section{Results and discussion Chemical syntheses}

The side chain of alkyl amines 5a-5g (Figures 1 and 2) was synthesized using the method we published previously. ${ }^{12}$ Starting from aromatic nitriles or aromatic aldehydes as raw material, aromatic tetrazoles $\mathbf{3}$ were synthesized by $(2+3)$ cycloaddition reaction with sodium azide, followed by reaction with phthalimide $\mathrm{N}$-alkyl bromides N-(3-bromopropyl) phthalimide, N-(4-bromo-butyl) phthalimide, or N-(5-bromopently) phthalimide to get $4 \mathrm{a}-4 \mathrm{~g}$, which

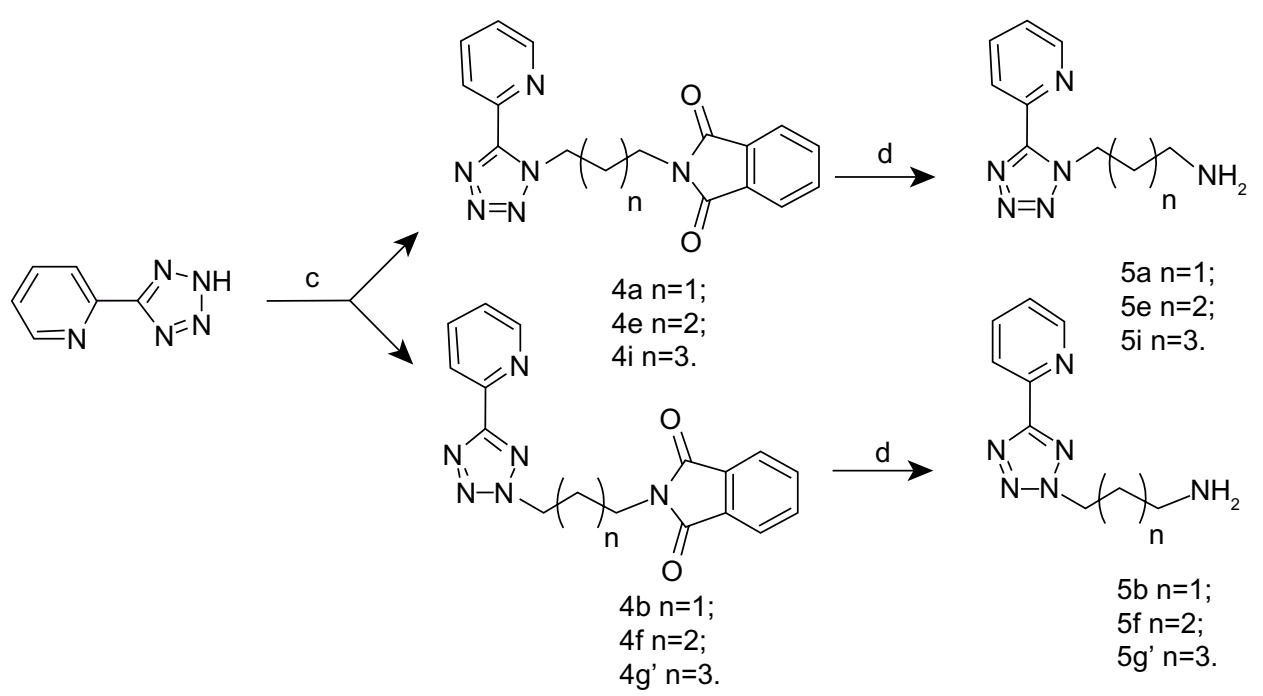

Figure 2 Synthesis of NI-submitted and N2-submitted 5-aryl-tetrazolyl alkylamines.

Notes: Reagents and condition: (c) $\mathrm{N}$-(3-bromopropyl)phthalimide, $\mathrm{N}$-(4-bromobutyl)phthalimide or $\mathrm{N}$-(5-bromopentyl)phthalimide, $\mathrm{K}_{2} \mathrm{CO}_{3}$, DMF, $80^{\circ} \mathrm{C} ;(\mathrm{d}) \mathrm{NH}_{2} \mathrm{NH}_{2} \cdot \mathrm{H}_{2} \mathrm{O}$, $\mathrm{EtOH} / \mathrm{CH}_{3} \mathrm{CN}$, reflux.

Abbreviations: DMF, N, N-dimethyl formamide; EtOH, ethanol. 
were then hydrazinolyzed to the desired tetrazole alkyl amine side chain.

Tanikawa et $\mathrm{al}^{14}$ synthesized similar acylide derivatives from clarithromycin by seven-step reactions in which selective removal of the $\mathrm{C}-3$ cladinose, protection of 2'-OH with acetyl, modification of the C-11,12 hydroxyl group by trichloromethyl chloroformate, acylation of the C-3 hydroxyl group, dehydrogenation of C-10,11, addition of carbonyl imidazole to $\mathrm{C}-12$, and then reaction with side chain amines were performed successively to get the $\mathrm{C}-11,12$ cyclic carbamates clarithromycin derivatives. Trichloromethyl chloroformate used in this routine has high toxicity and can cause serious health damage. Elliott et $\mathrm{al}^{15}$ improved the routine via six steps to synthesize a series of $\mathrm{C}-11,12$ cyclic carbamate acylide derivatives from clarithromycin. On the basis of the two classic methods, we have designed a new approach (Figure 3), less the reaction steps, in which the use of toxic trichloromethyl chloroformate could be avoided.

Treatment of clarithromycin with ethylene carbonate in refluxing triethylamine and vigorously stirring, triethylamine was distilled off, and then the cladinose was selectively removed under dilute aqueous acid to prepare compound 6; the 2'-hydroxyl group was protected with acetic anhydride, using dichloromethane as solvent to obtain compound 7 , followed by the esterification of 3-hydroxyl group with 3-pyridylacetic acid hydrochloride to yield compound 8 , in which the reaction must be carried out in real-time monitoring to prevent excessive reaction of esterification of the C-12 hydroxyl group. Compound 8, dissolved in anhydrous dichloromethane reacted with carbonyl diimidazole at room temperature, yielded the C-12 imidazolyl carbamate 9, followed by the reaction with excess side chain amines and then deprotection of the acetyl group by refluxing in methanol to yield the desired acylides (10a-10q) shown in Figure 4.

\section{Antibacterial activity}

The in vitro antimicrobial activities of acylides $10 \mathrm{a}-10 \mathrm{q}$ and standard antibiotics are shown in Table 1. The tabulated results show that all of the acylide derivatives $10 \mathrm{a}-10 \mathrm{q}$ exhibited potent antibacterial activity against the erythromycin-susceptible strains S. aureus ATCC 25923 and ATCC 6538, and most of them displayed excellent minimum inhibitory concentration values in the range of $0.06-0.5 \mu \mathrm{g} / \mathrm{mL}$, which is better than or comparable to erythromycin and clarithromycin, among which compounds $10 \mathrm{~g}$ and $10 \mathrm{o}$ were found to have the most potent activity against the erythromycin-susceptible strains tested. Compounds $10 \mathrm{f}$ and $10 \mathrm{~g}$ also exhibited excellent activity against $S$. epidermidis ATCC 12228.

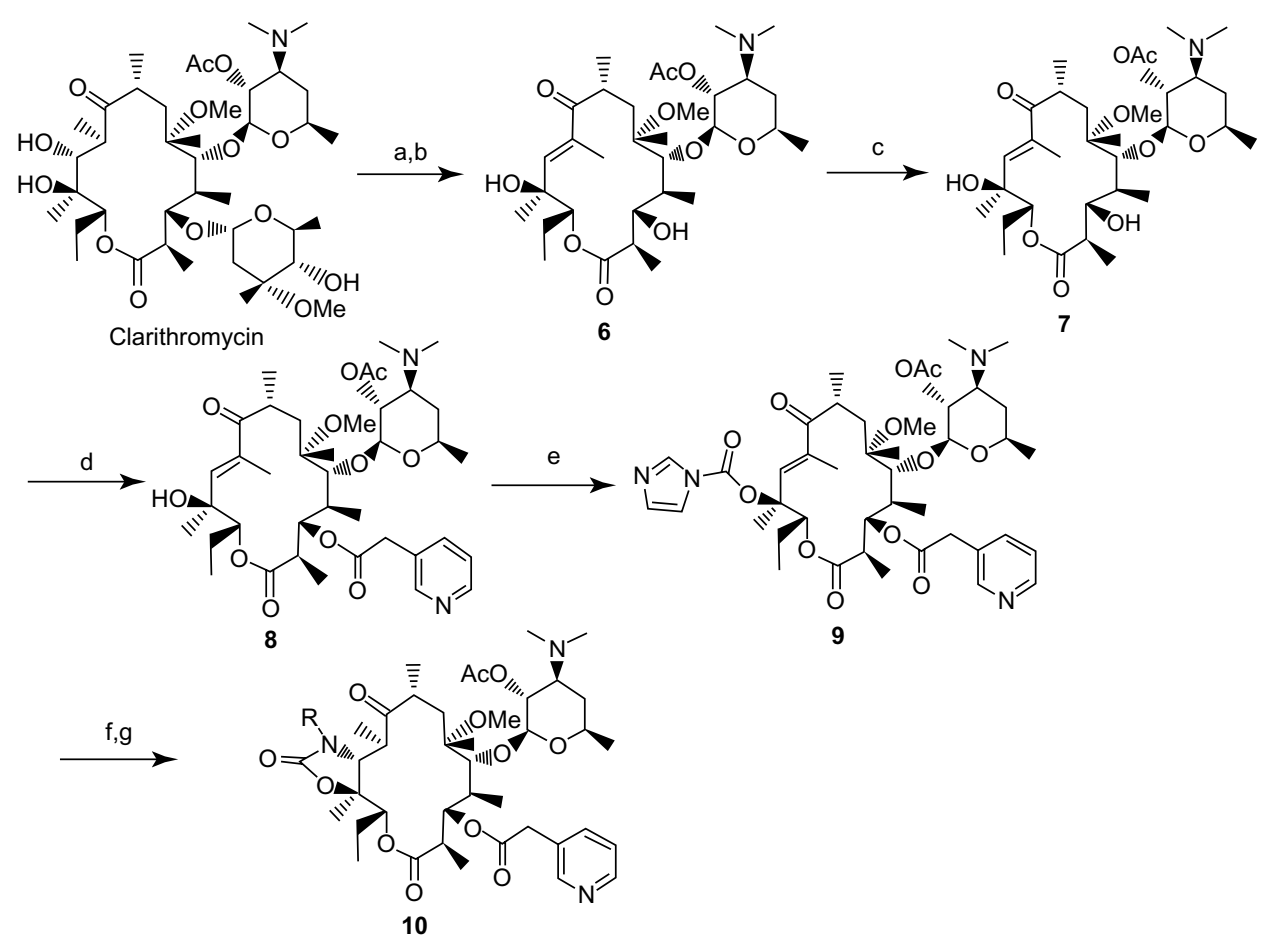

Figure 3 Synthesis of a set of acylide derivatives for compounds 10a- $10 \mathrm{~g}$.

Notes: Reagents and conditions: (a) Ethylene carbonate, TEA, reflux; (b) $\mathrm{HCl}, \mathrm{EtOH}, \mathrm{H}_{2} \mathrm{O}$; (c) $\mathrm{Ac}_{2} \mathrm{O}, \mathrm{TEA}_{2} \mathrm{CH}_{2} \mathrm{Cl}_{2}$; (d) $\mathrm{PivCl}_{2} \mathrm{DMAP}, \mathrm{CH}_{2} \mathrm{Cl}{ }_{2},-\mathrm{I} 5^{\circ} \mathrm{C}$ to rt; (e) $\mathrm{CDI}, \mathrm{DMAP}$, $\mathrm{CH}_{2} \mathrm{Cl}_{2}$, rt; (f) $\mathrm{RNH}_{2}, \mathrm{CH}_{3} \mathrm{CN} / \mathrm{H}_{2} \mathrm{O}, 55^{\circ} \mathrm{C}$; (g) $\mathrm{CH}_{3} \mathrm{OH}$, reflux.

Abbreviations: TEA, triethylamine; PivCl, pivaloyl chloride; EtOH, ethanol; CDI, carbonyl diimidazole; DMAP, 4-Dimethylaminopyridine; Ac, acetoxy; rt, room temperature. 


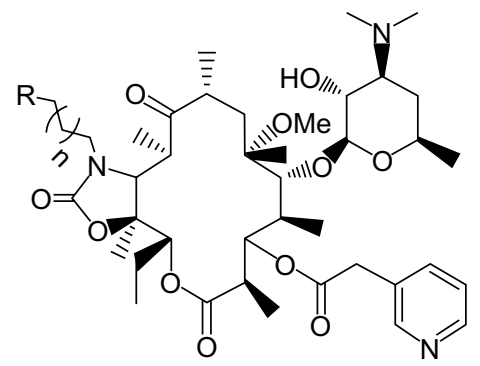

$(n=1 \sim 3)$
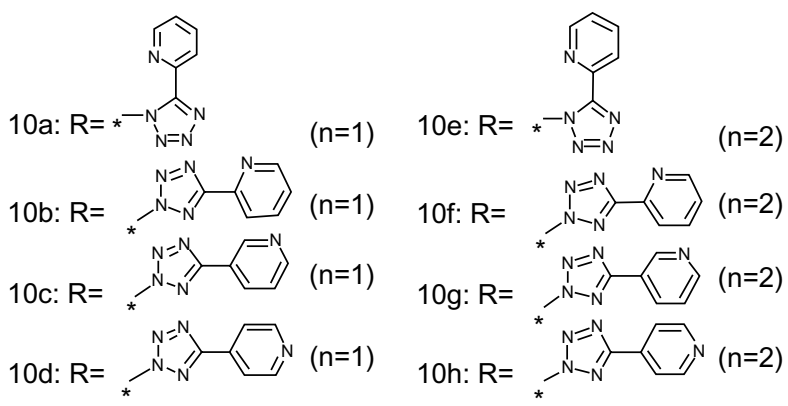

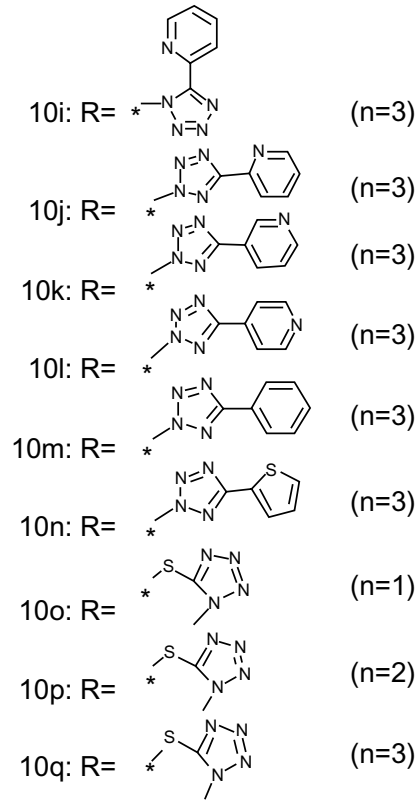

Figure 4 Structure synthesized acylides.

Note: *Connection position of $\mathrm{R}$ group to the parent compound.

Abbreviations: R, substituted group; Me, methyl.

Table I The antibacterial activities of novel acylides in vitro

\begin{tabular}{|c|c|c|c|c|c|c|c|}
\hline \multirow[t]{4}{*}{ Compound } & \multicolumn{7}{|c|}{ Minimum inhibitory concentration, $\mu \mathrm{g} / \mathrm{mL}$} \\
\hline & \multicolumn{2}{|c|}{ Staphylococcus aureus } & \multirow{3}{*}{$\begin{array}{l}\text { Staphylococcus } \\
\text { epidermidis } \\
\text { ATCC I } 2228\end{array}$} & \multicolumn{2}{|c|}{ Pseudomonas aeruginosa } & \multicolumn{2}{|c|}{ Escherichia coli } \\
\hline & ATCC 25923 & ATCC 6538 & & ATCC 1317 & ATCC 9027 & ATCC 8739 & ATCC 25922 \\
\hline & & & & & & & \\
\hline ERY & 0.5 & 0.5 & 0.5 & $>128$ & 128 & 32 & 8 \\
\hline CLA & 0.25 & 0.5 & 0.25 & 128 & 64 & 16 & 4 \\
\hline 10a & 0.25 & 1 & 0.125 & 32 & 64 & 16 & 16 \\
\hline $10 \mathrm{~b}$ & 0.25 & 0.25 & 0.25 & 128 & 32 & 32 & 64 \\
\hline $10 c$ & 1 & 0.5 & 0.25 & 128 & 128 & 64 & 64 \\
\hline $10 \mathrm{~d}$ & 0.5 & I & 0.5 & $>128$ & 128 & 64 & 64 \\
\hline $10 \mathrm{e}$ & 0.125 & 0.25 & 0.125 & 16 & 8 & 8 & 2 \\
\hline IOf & 0.125 & 0.125 & $<0.0625$ & 64 & 64 & 16 & 4 \\
\hline $\log$ & 0.125 & 0.063 & $<0.0625$ & 64 & 32 & 32 & 16 \\
\hline $10 \mathrm{~h}$ & 0.125 & 0.25 & 0.125 & 128 & 64 & 32 & 16 \\
\hline $10 \mathrm{i}$ & 0.25 & 0.5 & 0.125 & 128 & 64 & 64 & 32 \\
\hline $10 \mathrm{j}$ & 0.25 & 0.5 & 0.125 & 64 & 128 & 64 & 64 \\
\hline $10 \mathrm{k}$ & 0.5 & 0.5 & 0.25 & 128 & 64 & 64 & 64 \\
\hline $10 \mid$ & 0.5 & 0.25 & 0.5 & 128 & 128 & 128 & 64 \\
\hline $10 \mathrm{~m}$ & 0.125 & 0.5 & 0.5 & 128 & 64 & 64 & 64 \\
\hline $10 n$ & 0.125 & 0.5 & 0.5 & 64 & 32 & 16 & 16 \\
\hline 100 & $<0.0625$ & 0.125 & 0.25 & 16 & 16 & 8 & 4 \\
\hline $10 p$ & 0.125 & 0.125 & 0.25 & 64 & 32 & 32 & 32 \\
\hline $10 q$ & 0.25 & 0.25 & 0.25 & 128 & 128 & 128 & 64 \\
\hline
\end{tabular}

Abbreviations: ATCC, American Type Culture Collection; ERY, erythromycin; CLA, clarithromycin.

Some of the acylide derivatives showed slightly more potent activity against $P$. aeruginosa and E. coli. Compounds $10 \mathrm{e}$ and $10 \mathrm{o}$ seemed to be more potent than other tetrazole-containing acylide derivatives against P. aeruginosa ATCC 9027 and ATCC 1317. Compared with erythromycin, compound $10 \mathrm{e}$ and compound $10 \mathrm{o}$ also exhibited improved potencies against E. coli strains.

\section{Conclusion}

Seventeen acylide derivatives have been synthesized and evaluated for in vitro antibacterial activities against 
Gram-positive and Gram-negative pathogens. All of them were found to be potent against the strains. In particular, the compounds $10 \mathrm{e}-10 \mathrm{~h}$, with a long side-chain alkyl having four carbon atoms, exhibited better antibacterial activities against erythromycin-susceptible strains. Compound 100 with a side chain of 3 carbon atoms and a sulfur atom also showed better antibacterial activity against the five strains.

\section{Acknowledgments}

This work was supported by the National Natural Science Foundation of China (No 81072554, 81373285) and by the 111 Project (No B13038).

\section{Disclosure}

The authors report no conflicts of interest in this work.

\section{References}

1. Song JH, Baek JY, Cheong HS, Peck KR, Ko KS. Incorrect identification of Streptococcus pneumoniae and its effect on antimicrobial resistance rates. Int J Antimicrob Agents. 2009;33(1):93-95.

2. Zhanel GG, Dueck M, Hoban DJ, et al. Review of macrolides and ketolides: focus on respiratory tract infections. Drugs. 2001;61(4):443-498.

3. Ma Z, Nemoto PA. Discovery and development of ketolides as a new generation of macrolide antimicrobial agents. Curr Med Chem Anti-Infect Agents. 2002;1(1):15-34.

4. Brvar M, Perdih A, Hodnik V, et al. In silico discovery and biophysical evaluation of novel 5-(2-hydroxybenzylidene) rhodanine inhibitors of DNA gyrase B. Bioorg Med Chem. 2012;20(8):2572-2580.
5. Allen NE. Macrolide resistance in Straphylococcus aureus: inducers of macrolide resistance. Antimicrob Agents Chemother. 1977;11(4): 669-674.

6. Tanikawa T, Asaka T, Kashimura M, et al. Synthesis and antibacterial activity of acylides (3-O-acyl-erythromycin derivatives): a novel class of macrolide antibiotics. J Med Chem. 2001;44(24):4027-4030.

7. Abell AD. Heterocyclic-based peptidomimetics. Lett Pept Sci. 2002;8(3-5):267-272.

8. Myznikov LV, Hrabalek A, Koldobskii GI. Drugs in the tetrazole series. Chem Heterocycl Compd. 2007;43(1):1-9.

9. Hayashi R, Jin X, Cook GR. Synthesis and evaluation of novel heterocyclic MMP inhibitors. Bioorg Med Chem Lett. 2007;17(24): 6864-6870.

10. Lebreton L, Curet O, Gueddari S, et al. Selective and potent monoamine oxidase type B inhibitors: 2-substituted 5-aryltetrazole derivatives. J Med Chem. 1995;38(24):4786-4792.

11. Adamec J, Beckert R, Weiss D, et al. Hybrid molecules of estrone: new compounds with potential antibacterial, antifungal, and antiproliferative activities. Bioorg Med Chem. 2007;15(8):2898-2906.

12. Song QL, Guo BQ, Zhang W, Lan P, Sun PH, Chen WM. Design, synthesis and antibacterial activity of novel ketolides bearing an aryltetrazolylsubstituted alkyl side chain. J Antibiot (Tokyo). 2011;64(8):571-581.

13. Herr RJ. 5-Substituted-1H-tetrazoles as carboxylic acid isosteres: medicinal chemistry and synthetic methods. Bioorg Med Chem. 2002;10(11):3379-3393.

14. Tanikawa T, Asaka T, Kashimura M, et al. Synthesis and antibacterial activity of a novel series of acylides: 3-O-(3-pyridyl)acetylerythromycin A derivatives. $J$ Med Chem. 2003;46(13):2706-2715.

15. Elliott RL, Pireh D, Griesgraber G, et al. Anhydrolide macrolides. 1. Synthesis and antibacterial activity of 2,3-anhydro-6-O-methyl 11,12carbamate erythromycin A analogues. J Med Chem. 1998;41(10): $1651-1659$.

\section{Publish your work in this journal}

Drug Design, Development and Therapy is an international, peerreviewed open-access journal that spans the spectrum of drug design and development through to clinical applications. Clinical outcomes, patient safety, and programs for the development and effective, safe, and sustained use of medicines are a feature of the journal, which has also been accepted for indexing on PubMed Central. The manuscript management system is completely online and includes a very quick and fair peer-review system, which is all easy to use. Visit http://www.dovepress.com/testimonials.php to read real quotes from published authors. 